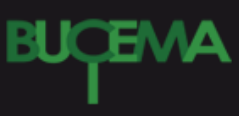

Bulletin du centre d'études médiévales d'Auxerre | BUCEMA

Hors-série $n^{\circ} 12$ | 2020

De la clôture à la fortification des monastères

\title{
Barrières, clôtures et murailles à Landévennec : juxtaposition et évolution
}

Ronan Pérennec et Annie Bardel

\section{OpenEdition}

1 Journals

Édition électronique

URL : https://journals.openedition.org/cem/17352

DOI : 10.4000/cem.17352

ISSN : 1954-3093

Éditeur

Centre d'études médiévales Saint-Germain d'Auxerre

Référence électronique

Ronan Pérennec et Annie Bardel, «Barrières, clôtures et murailles à Landévennec : juxtaposition et évolution », Bulletin du centre d'études médiévales d'Auxerre | BUCEMA [En ligne], Hors-série n 12 | 2020, mis en ligne le 25 novembre 2020, consulté le 04 mars 2023. URL : http://journals.openedition.org/ cem/17352 ; DOI : https://doi.org/10.4000/cem.17352

Ce document a été généré automatiquement le 4 mars 2023

Creative Commons - Attribution - Pas d'Utilisation Commerciale - Partage dans les Mêmes Conditions 4.0 International - CC BY-NC-SA 4.0

https://creativecommons.org/licenses/by-nc-sa/4.0/ 


\title{
Barrières, clôtures et murailles à Landévennec : juxtaposition et évolution
}

\author{
Ronan Pérennec et Annie Bardel
}

1 La clôture, qui isole les moines du siècle, est un des fondements d'un monastère. Mais le principe s'est perfectionné au cours du temps: l'ancienne abbaye de Landévennec, située à la pointe de la Bretagne, est tout à fait représentative en ce domaine. Entre sa fondation, aux environs de l'an 500, et son abandon après 1789 , elle a vu évoluer considérablement les systèmes de cloisonnement qui régissaient son organisation.

2 La confrontation des sources écrites et des données archéologiques a permis d'appréhender en grande partie les différents niveaux de clôture dès le haut Moyen Âge. On peut ainsi distinguer : le domaine monastique, pris dans un sens plus ou moins large, la monachia, ensemble des lieux réguliers, le claustrum ou le cloître, mais aussi le castrum, enceinte défensive et, dans un contexte plus restreint, les aménagements conçus dans l'église pour limiter l'accès ou la circulation dans ce lieu public.

3 La spécification et la complexité des divers dispositifs s'étant affirmées au fil du temps, leur présentation s'inscrit naturellement dans une suite chronologique.

\section{Une clôture naturelle}

4 Concernant le domaine monastique, plusieurs publications ont déjà traité des nombreuses possessions de l'abbaye ${ }^{1}$. Il n'y a pas lieu d'y revenir ici et nous ne nous intéresserons donc qu'au domaine proche. L'abbaye de saint Guénolé est installée au pied de la «montagne» de Penforn. Ce qualificatif un peu pompeux, eu égard à la géographie locale, est utilisé dès le Ix siècle sous la plume de l'hagiographe Gurdisten². Il reflète une réalité concrète : l'existence de reliefs bien marqués, notamment dans tout le secteur occidental. La pointe de Penforn, qui représente le domaine proche, se présente comme une langue de terre bordée sur trois côtés par la ria de l'Aulne (fig. 1). La disposition des lieux n'est pas sans rappeler un site d'oppidum, mais une éventuelle 
occupation protohistorique de cette fortification naturelle reste à prouver. Si l'accès en était barré, les vestiges ne sont pas repérables à cause des nombreux remaniements qui ont affecté le terrain par la suite.

Fig. 1 - Localisation de Landévennec et vue aérienne de la pointe de Penforn

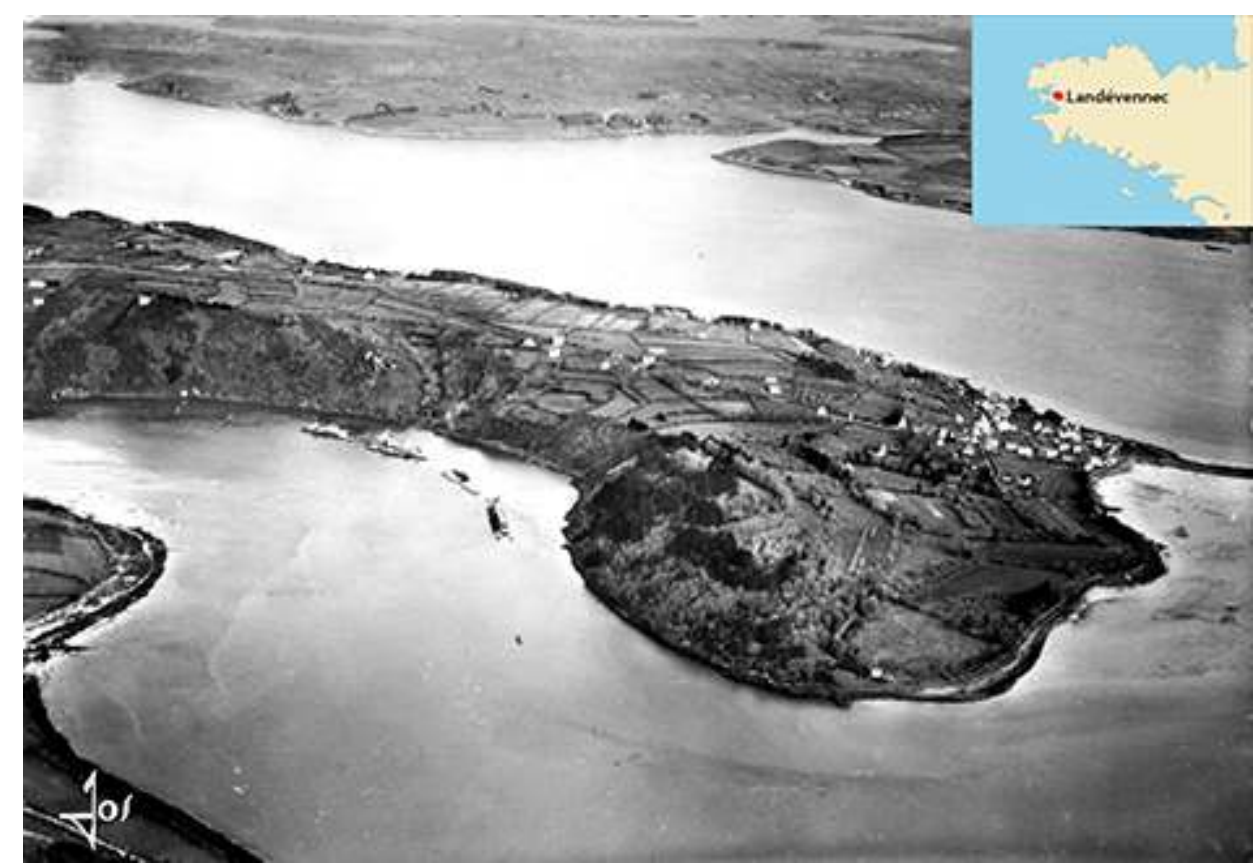

(Cl. Jos Le Doaré)

5 On ne sait donc pas si l'enceinte du haut Moyen Âge a repris ou non un tracé préexistant de ce côté. En revanche, comme sur l'îlot de Tibidy qui accueillit le premier établissement de Guénolé, la clôture sur les trois autres côtés était naturelle et maritime, étant donné la disposition même de la pointe de Penforn. Il semblerait que l'esprit défensif ne présidait pas à l'installation des premiers bâtiments monastiques. Ils ont été implantés sur l'emplacement le plus accessible et le plus ouvert, sur le côté oriental de la pointe, là où un ruisseau avait tracé une vallée en forme d'amphithéâtre (fig. 2). La pente moyenne, ajoutée à la présence d'eau, pouvait convenir à la création d'un clos agricole. La frange forestière sur son pourtour, où le relief trop abrupt, ne permettait pas une mise en culture, délimitait et complétait à la fois cet espace ${ }^{3}$. 
Fig. 2 - Localisation du site par rapport au relief du domaine de Penforn

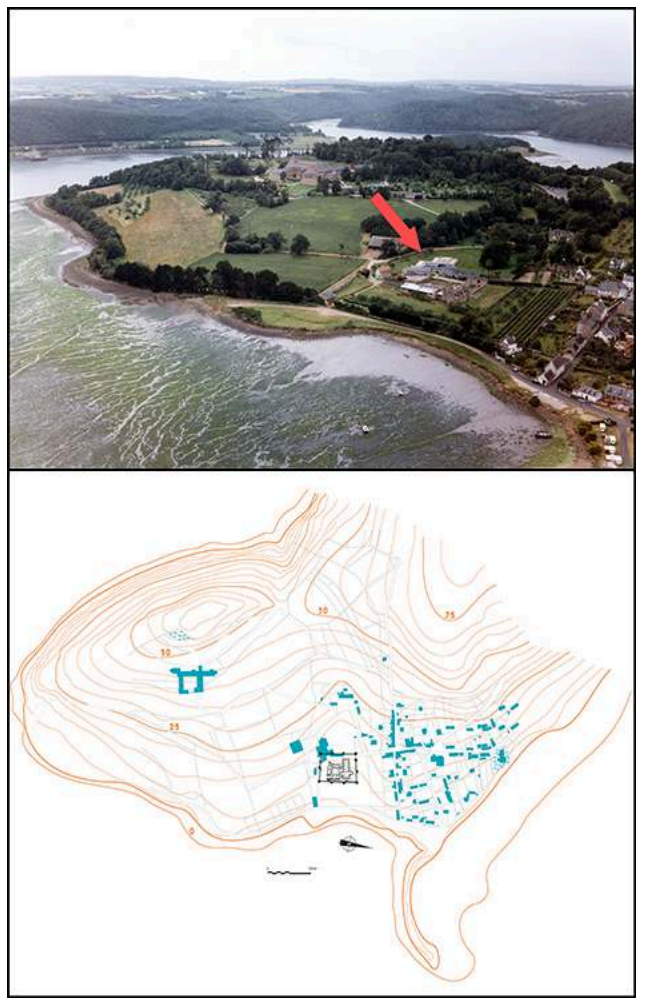

(Cl. P. Martin; dessin V. Bardel)

6 Il est indispensable de signaler que les plus anciens vestiges repérés sur le site sont ceux d'un bâtiment arasé entouré d'un cimetière. Ils sont datés de la charnière des ve$\mathrm{VI}^{\mathrm{e}}$ siècles $^{4}$ (fig. 3). Pour résumer, précisons que le rapport stratigraphique direct établi dès les origines entre les sépultures et le bâti a permis d'identifier un oratoire. Il faut y ajouter le fait que cette structure est restée une chapelle, à travers six reconstructions successives et sur une durée de treize siècles ${ }^{5}$. 
Fig. 3 - Restitution partielle de l'oratoire et du cimetière primitifs avec le cours d'eau central

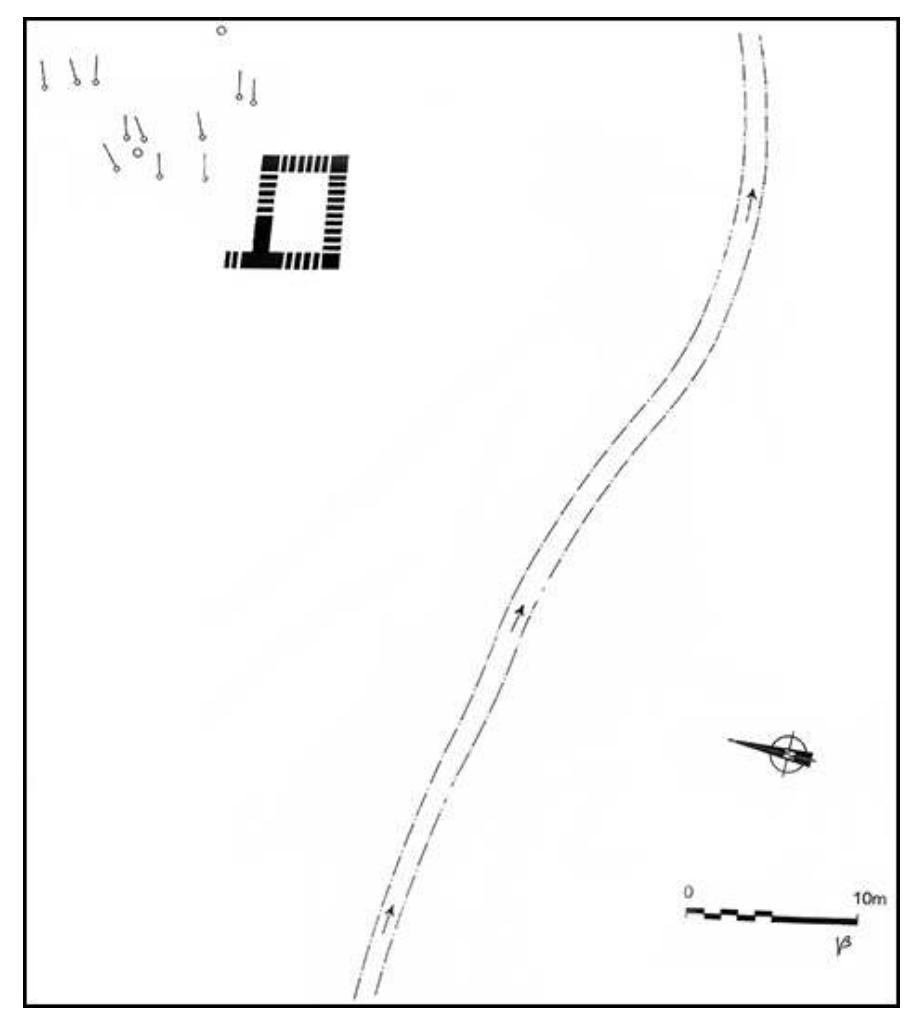

(DAO V. Bardel)

7 Si l'on excepte celui qui est daté par ${ }^{14} \mathrm{C}$, les squelettes figurant sur le plan de la fig. 3 sont donnés à titre indicatif, dans la mesure où ils ne sont datables formellement qu'à l'intérieur d'une période couvrant les $\mathrm{VI}^{\mathrm{e}}$ et $\mathrm{VII}^{\mathrm{e}}$ siècles. L'affleurement du substrat rocheux compacte les relations stratigraphiques et, s'il est évident de raccorder les tombes, surtout les plus anciennes, avec le bâtiment, il n'est guère possible de fixer la limite supérieure de cette période. D'un autre côté, le recensement est incomplet: les sépultures qui n'avaient pas été détruites ou occultées par les structures postérieures ont disparu en partie au cours de fouilles anciennes.

Il est donc impossible d'évaluer le nombre d'occupants du cimetière au vie siècle.

8 Pour cette période, on ne connaît pas non plus l'emplacement du tout premier habitat monastique qui, selon la Vita, y était associé. La présence même d'un habitat est controversée. Or Gurdisten emploie le terme «fundus » pour désigner le lieu où se sont fixés Guénolé et ses compagnons ${ }^{6}$. Il s'agit donc d'un domaine cultivé et il est situé à proximité d'une villa ${ }^{7}$. La donation implique logiquement une volonté de poursuite ou de reprise de l'exploitation par un groupe humain. L'habitat proprement dit, qui semble traditionnellement séparé à cette époque des lieux d'inhumation ${ }^{8}$, a pu s'installer tout d'abord dans des bâtiments désaffectés, cédés avec les terres, et s'établir ensuite à côté du lieu de culte. Sachant qu'un des points essentiels de la légende fondatrice raconte le déplacement du monastère "d'un jet de pierres vers l'est », il est concevable qu'elle rende compte de cette évolution. Mais quelles que soient les conjectures faites à ce sujet, elles ne permettent pas de résoudre la question de la disposition ni, à plus forte raison, celle des limites du domaine primitif. 
9 En ce qui concerne l'espace religieux proprement dit, on n'a pas retrouvé de traces d'une clôture, ce qui est concevable, mais ne peut être considéré comme décisif, étant donné l'empilement des structures postérieures.

Les premières traces d'habitat sur le site même apparaissent à la fin $\mathrm{du} \mathrm{VI}^{\mathrm{e}} \mathrm{ou}$ au début $\mathrm{du} \mathrm{VII}^{\mathrm{e}}$ siècle $^{9}$, à une époque où l'on constate que les vivants viennent rejoindre les morts ${ }^{10}$. Elles sont concentrées sur une aire intercalée entre l'oratoire et le ruisseau. Contrairement à ce que l'on aurait attendu, les données archéologiques ne laissent pas entrevoir des cellules dispersées, mais plutôt un bâti de type longère, dont on ne connaît cependant pas l'emprise exacte ni le mode de construction (fig. 4). Celui-ci semble regrouper toutes les fonctions d'un habitat monastique, mais la présence d'annexes plus ou moins proches, dont les vestiges auraient été enfouis sous les structures plus récentes, ne doit pas être exclue, là encore.

Fig. 4 - Restitution de l'oratoire et des premières traces d'habitat

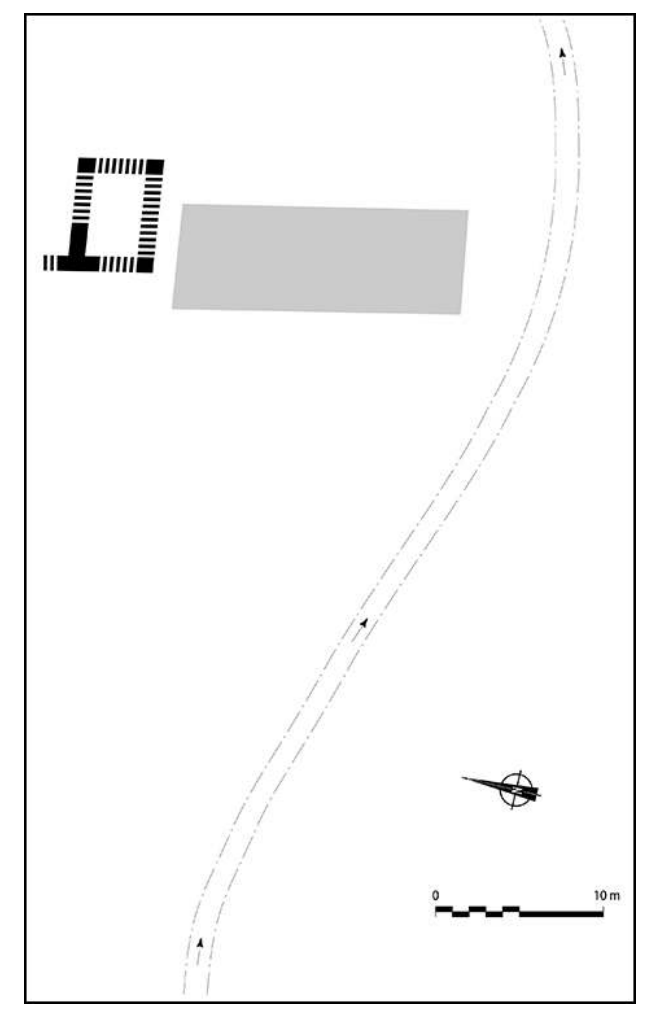

(DAO V. Bardel)

11 Le monastère primitif n'était pas nécessairement clos. Pourtant, si l'on suit la légende de saint Guénolé, il serait logique d'envisager l'existence d'un domaine fermé ; deux indices dans sa Vita semblent l'attester. Le premier fait écho d'un miracle à ce sujet : afin d'éviter que les moines n'aient à sortir du monastère pour aller chercher de l'eau, le saint fait jaillir une source à l'intérieur du "cloître " ${ }^{11}$. De même, l'épisode des voleurs qui, arrivés en barque, se sont introduits de nuit à l'intérieur de l'enceinte, suggère a minima la présence de palissades, bien qu'aisément franchissables ${ }^{12}$.

12 Notons toutefois que ces faits sont rapportés par Gurdisten, quasiment trois siècles plus tard, à une époque où la notion de clôture est indubitable. Et outre, une Vita retranscrivant des codes établis et ne pouvant donc être prise au pied de la lettre, il 
faut considérer que l'enceinte qu'évoque Gurdisten est très certainement celle qu'il connaît - ou qu'il extrapole. Et même si la délimitation est d'origine ancienne, rien ne nous indique de quelle façon elle était matérialisée.

\section{Un enclos maçonné pour la monachia}

La première clôture archéologiquement repérable est datée du milieu du VII siècle $^{13}$. Elle est incomplète, mais selon toute apparence, elle isole dans un enclos l'ensemble constitué par l'oratoire, le cimetière et l'habitat, qui apparaissent dès lors comme la monachia, espace de vie monastique distinct du domaine proche. La fermeture était assurée par une maçonnerie dont on a mis au jour une partie des vestiges très arasés, du côté de la mer (fig. 5). Il en reste un solin de pierres liées à l'argile, d'une largeur de $0,80 \mathrm{~m}$, que l'on a interprété tout d'abord comme le support d'une palissade de bois. Le fait que les assises de pierres soient posées sur le substrat rocheux, sans fondation, ne semble cependant pas déterminant et n'exclut pas en principe l'hypothèse d'un mur en élévation, que justifierait la largeur de la base conservée ${ }^{14 .}$

Cette clôture était percée d'un seuil en escalier, large de 1,80 m, montant par trois marches vers l'intérieur de l'enclos. Il est à remarquer que cette porte était située juste en face du chevet de l'oratoire. Celui-ci constituait à l'époque la seule église et renfermait le tombeau de saint Guénolép ${ }^{15}$.

Fig. 5 - Vestiges de la clôture orientale des $\mathrm{VII}^{\mathrm{e}}$ et VIII siècles et de son seuil

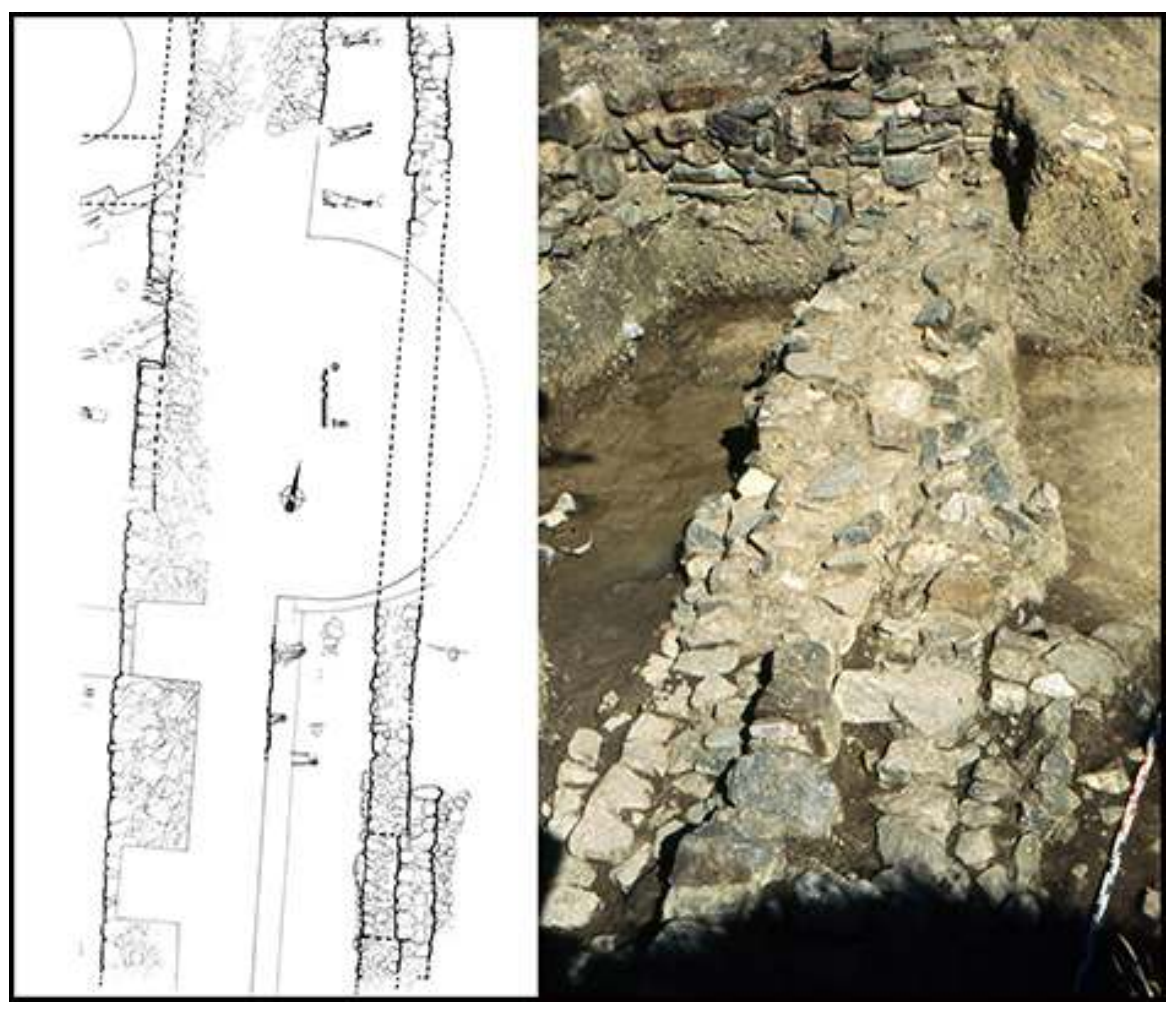

(Cl. des auteurs; DAO V. Bardel)

On ne connaît pas les prolongements de ce mur sur le pourtour du monastère. Représentait-il une enceinte continue? C'est tout à fait probable si l'on se fie aux 
quelques données disponibles pour les époques postérieures. Mais quelle était la surface enclose? Il n'est possible de reconstituer ni son tracé, ni son mode de construction sur le reste du périmètre.

\section{Un second niveau de clôture : le claustrum}

Dans le même temps, vers 662, le bâtiment unique accolé à l'oratoire est complété par une construction sur poteaux indépendante, installée sur la rive sud du cours d'eau, alors que l'ensemble monastique était concentré jusqu'à présent sur le versant nord. Un alignement de trois poteaux de bois dont les bases se sont conservées en place, indique que ce bâti était disposé le long du ruisseau, selon un axe grossièrement perpendiculaire à l'aile orientale (fig. 6) ${ }^{16}$. La disposition qui est alors mise en place va perdurer, en dépit de réfections et réaménagements, jusqu'au milieu du IX ${ }^{e}$ siècle.

Fig. 6 - Le monastère vers 662 : plan et restitution partielle

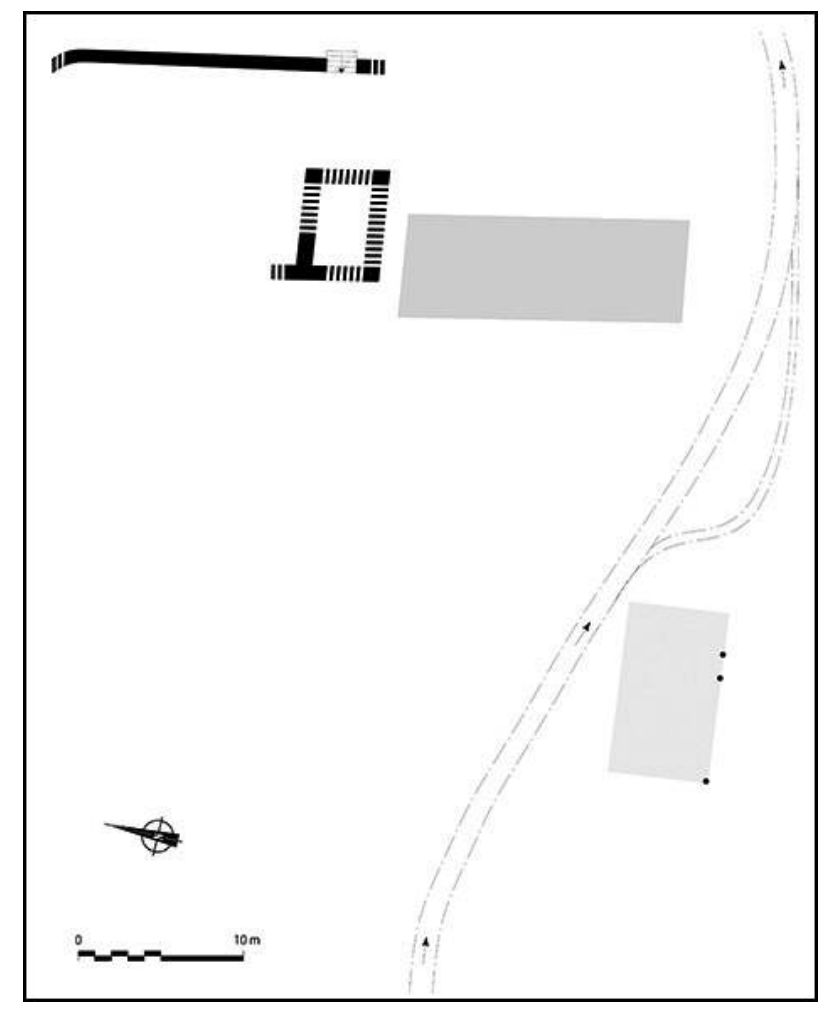

(DAO V. Bardel)

17 L'agrandissement progressif des deux ailes de bâtiments au cours des $\mathrm{VII}^{\mathrm{e}}$ et $\mathrm{VIII}^{\mathrm{e}}$ siècles détermine la création d'une cour, traversée en diagonale par le ruisseau (fig. 7). Bien qu'elle ne soit pas entièrement cernée par des édifices et qu'elle reste donc ouverte en grande partie, cette cour, par sa position centrée, constitue toutefois un espace de circulation interne, par opposition au pourtour extérieur des bâtiments.

18 Fig. 7 - Plan et restitution partielle du monastère à la fin du viI ${ }^{\mathrm{e}}$ siècle (1) et au milieu du $\mathrm{VIII}^{\mathrm{e}}(2)$ 


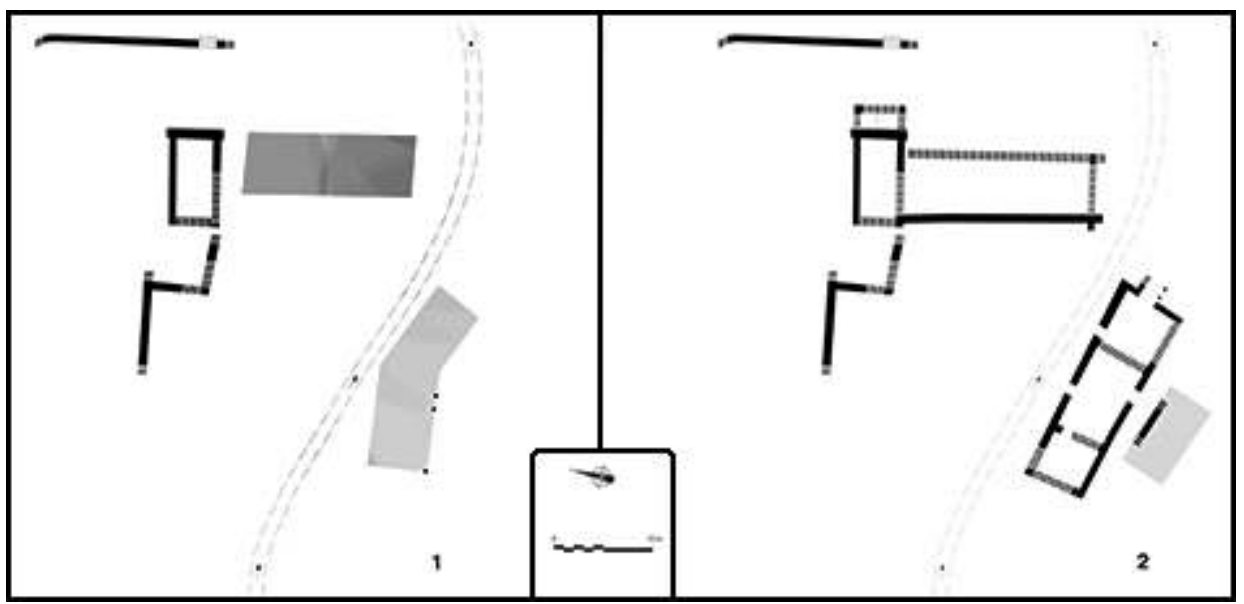

(DAO V. Bardel)

Ce statut différent est marqué par la construction d'une clôture supplémentaire, qui vient fermer la cour du côté de l'oratoire tout en délimitant un parvis devant son portail ${ }^{17}$. Le lieu de culte est lui-même reconstruit et agrandi. Il est logique de supposer que les moines s'isolaient ainsi des laïcs, qui fréquentaient sans doute l'église en nombre grandissant.

De cette clôture, on n'a retrouvé, là encore, que quelques assises d'un mur ou d'un solin de pierres, ce qui ne permet pas de déterminer s'il s'agissait d'un mur ou d'une palissade. Les structures postérieures, mais aussi la remontée du terrain vers l'ouest, ont fait disparaître la suite de cette maçonnerie, très arasée. Mais on peut imaginer un prolongement en retour qui aurait achevé d'enclore cet espace central, côté ouest. Cette disposition est hypothétique et ne représente pour lors qu'une extrapolation, mais on sait qu'elle a été adoptée au IX ${ }^{\mathrm{e}}$ siècle pour enclore la même cour.

21 Durant toute cette période, la barrière extérieure repérée du côté de la mer reste en place, sans changement visible. On voit donc apparaître dès ce moment deux niveaux de clôtures: la première devait entourer l'ensemble des bâtiments monastiques, la seconde délimiterait un claustrum, espace réservé aux moines à l'exclusion probable des laïcs.

\section{Des délimitations plus visibles et différenciées}

$2 \mathrm{Au}$ cours du IX ${ }^{\mathrm{e}}$ siècle, le monastère évolue en établissement bénédictin. En ce qui concerne le bâti, cette mutation se fait en deux temps. Dans les débuts du siècle, une église de plan basilical est tout d'abord édifiée au nord de l'oratoire, qui devient une chapelle annexe $\mathrm{e}^{18}$. Dans la seconde moitié du siècle, c'est le monastère dans son ensemble qui est reconstruit selon un plan orthonormé (fig. 8). 
Fig. 8 - 1) Plan du monastère carolingien au début du IXe siècle. 2) Plan vers 870 après reconstruction des bâtiments ; le chœur a été réaménagé pour accueillir les reliques de saint Guénolé

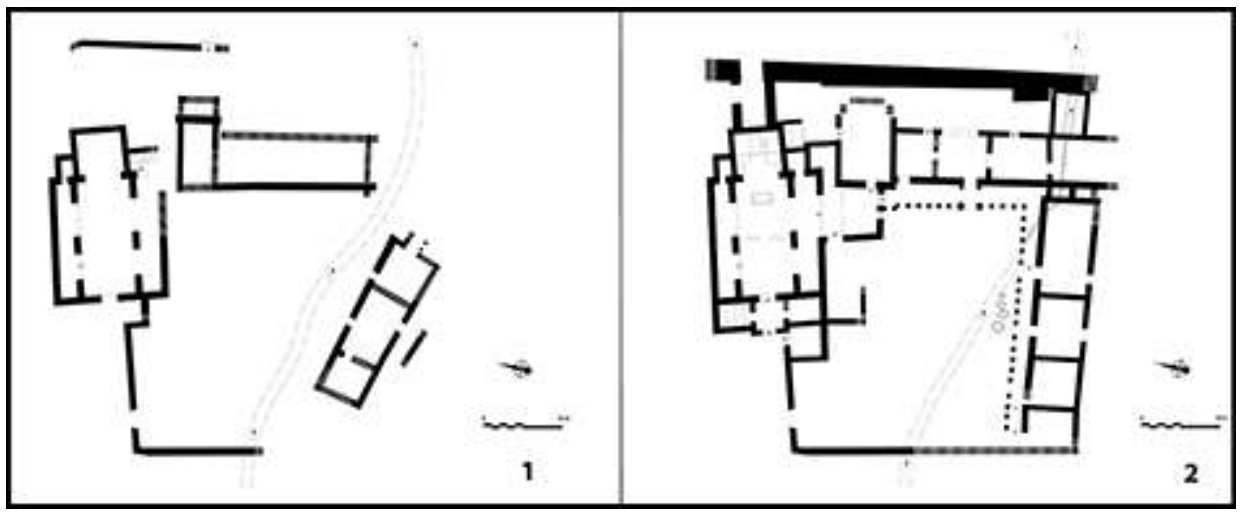

(DAO V. Bardel)

24 Cette évolution s'accompagne de changements marquants et aboutit à une plus grande diversité des types de cloisonnements. On pourrait considérer que ces transformations ne sont qu'apparentes dans la mesure où la meilleure conservation de ces niveaux a pu occulter, on l'a dit, une partie des structures antérieures. Mais il s'avère toutefois que les démarcations sont plus affirmées et différenciées que précédemment et qu'elles révèlent des zones non identifiées jusqu’à présent.

\section{Un enclos monastique}

Les limites de la clôture monastique globale ne sont pas connues pour le haut Moyen Âge et on ne dispose pas de données archéologiques suffisantes, ni de références écrites permettant de reconstituer l'ensemble du pourtour. Mais on a vu plus haut que Gurdisten mentionnait une source - d'origine miraculeuse - qu'il place à l'intérieur de l'enceinte. Même si elle ne peut pas être appliquée d'office aux monastères qui l'ont précédé, cette mention de Gurdisten donne une indication précieuse sur l'extension de l'enclos dans ce secteur à l'époque carolingienne, dans la mesure où la source est encore en place actuellement sous la forme d'une fontaine, à une centaine de mètres à l'ouest du monastère.

26 À partir de là, des limites possibles de l'enceinte semblent conservées en place et fixées par les plans cadastraux, qui se calquent en grande partie sur deux séries de talus (fig. 9). 
Fig. 9 - Restitution sur fond cadastral du double enclos monastique du haut Moyen Âge avec la fontaine (a) et la chapelle (b)

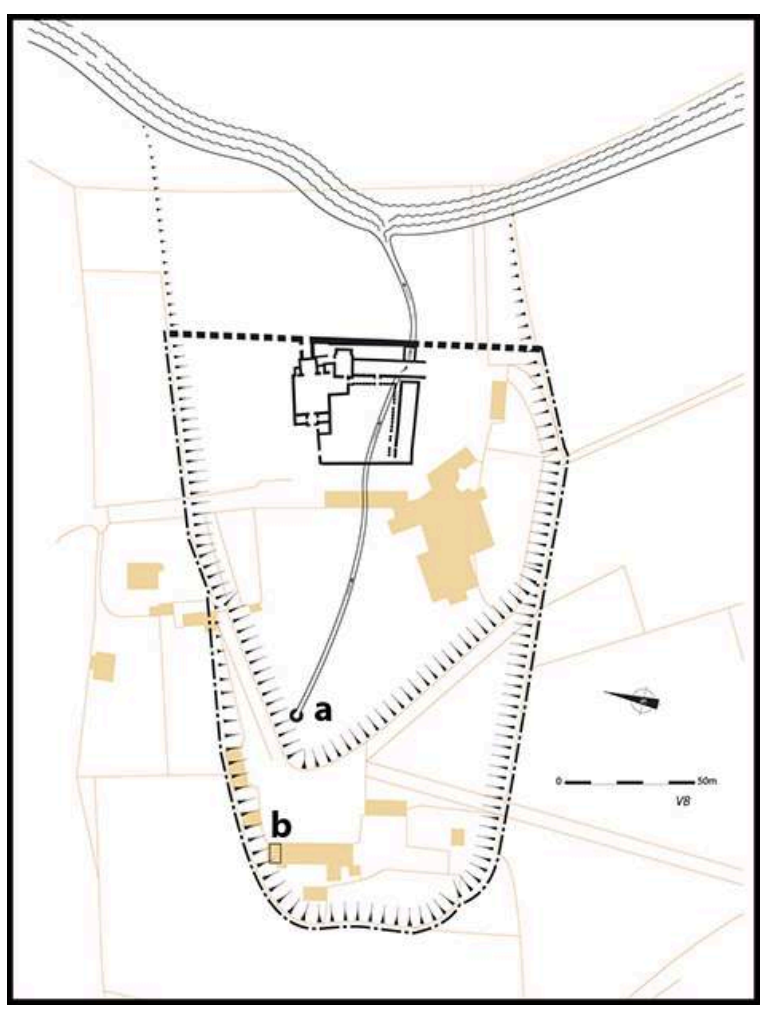

(DAO V. Bardel)

On obtient deux tracés imbriqués, adaptés au relief originel du terrain (fig. 2). Il faut tenir compte cependant du fait que cette disposition, de même que la terrasse supérieure encore en place actuellement, résulte en partie d'aménagements importants du terrain naturel qui se sont répartis dans le temps et qu'il est difficile de dater. Les structures conservées jusqu'à nous sont en partie indéterminables, d'époque moderne ou de la fin du Moyen Âge. Les levées de terre atteignent, côté ouest, entre 2 et $3 \mathrm{~m}$ de hauteur, mais s'amenuisent nettement vers la mer. De très larges portions en subsistent encore, même si par endroits, seule la trace au sol du talus arasé est visible. Enfin, certaines parties ont été reprises en pierres, dont une porte avec échaliers ouverte dans un talus et donnant sur la métairie.

Mais la conformité des tracés avec des constructions anciennes - une fontaine et une chapelle en l'occurrence -, permet de considérer que dans leur premier état, ils ont été implantés au haut Moyen Âge. En effet, le contour le plus restreint, d'une longueur totale de $162 \mathrm{~m}$ sur $150 \mathrm{~m}$ de largeur à sa base, côté mer, englobe le monastère et se termine en triangle vers l'ouest pour entourer précisément la fontaine. L'autre enceinte, dont la base est la même, forme un quadrilatère de $100 \mathrm{~m}$ de large qui s'étend vers l'ouest jusqu'à $230 \mathrm{~m}$ de long, pour enserrer le lieu-dit «Le Penity », devenu le manoir des abbés commendataires. Ce lieu avait été occupé auparavant par une chapelle dédiée à saint Vallée (Guénolé), construite au XV siècle et détruite au XVIII par l'extension du manoir. Malgré cette datation tardive, ce lieu de culte avait, selon la tradition, des origines plus anciennes, puisque son toponyme est considéré comme remontant au $\mathrm{x}^{\mathrm{e}}$ siècle $^{19}$. Des sondages pratiqués sur cet emplacement à l'occasion de travaux de réhabilitation du bâtiment n'ont amené la mise au jour d'aucun vestige 
antérieur, mais cela n'est pas vraiment significatif dans la mesure où les investigations ont été très restreintes par les travaux en cours.

La proximité entre chapelle et source et le fait qu'elles soient incluses dans l'extrémité ouest de l'enclos supposé pourraient donc être considérés comme une confirmation de ce tracé. Il aurait, dans ce cas, prolongé à l'époque moderne des limites établies de tout temps. Mais si elles remontent vraiment au haut Moyen Âge, cela n'implique pas que les deux enceintes soient contemporaines. Le plus grand tracé, lié à la chapelle, pourrait sous toutes réserves être le plus récent, d'époque carolingienne ou même postérieur ${ }^{20}$. Tandis que l'enceinte la plus réduite, dans la mesure où elle englobe la source, pouvait même être associée à la clôture mise au jour à l'est et datée du milieu du $\mathrm{VII}^{\mathrm{e}}$ siècle. Si c'était le cas, auraient-elles représenté la même limite ou deux cloisonnements imbriqués et donc distincts ? Le fait que les talus semblent se prolonger jusqu'à la grève, au-delà du mur oriental, indiquerait plutôt deux espaces imbriqués : la monachia, délimitée par un mur, à l'intérieur d'un enclos plus large. Dans cette hypothèse, nous aurions alors une indication supplémentaire sur le système de clôture pour les périodes les plus anciennes.

Quoi qu'il en soit, et quelles que soient ses origines, du point de vue de l'organisation du monastère, cet enclos monastique se situe entre le domaine proche, représenté par la pointe de Penforn, et la monachia, définie comme le lieu de vie spirituel. Il représente un espace intermédiaire, comprenant manifestement des jardins et cultures, séparé lui aussi du monde extérieur grâce à une démarcation spécifique.

\section{Un castrum}

31 Dans quelle mesure est-il distinct du castrum, dont les aménagements sont décrits également par Gurdisten? Indépendamment des limites domaniales ou monastiques, l'abbaye possédait en effet une enceinte à vocation défensive. Celle-ci ne s'affirme clairement qu'à partir du ix siècle, sous la menace des raids scandinaves. Elle se concentre alors du côté de la mer. Gurdisten précise en effet que son monastère est clos de ce côté par un mur dans lequel s'ouvre l'unique porte d'accès et que le reste du pourtour est protégé par des talus et fossés, puisqu'il s'appuie du côté ouest "sur les contreforts boisés des montagnes" qui rendent l'accès difficile ${ }^{21}$.

La documentation archéologique fait défaut pour appréhender dans son intégralité la fortification carolingienne. Les recherches n'ont porté que sur le secteur oriental et ont pu y mettre en évidence, à côté de la clôture primitive, les bases d'un large mur qui fermait le monastère de ce côté $\mathrm{au} \mathrm{IX}^{\mathrm{e}}$ siècle, seule limite qui soit donc connue actuellement (fig. 8) ${ }^{22}$.

En ce qui concerne le reste des ouvrages défensifs, les mêmes problèmes que ceux rencontrés précédemment se posent quant à leur implantation. Si le secteur ouest est protégé par les contreforts montagneux, son étendue le rend peu propice à la défense. Il serait plus raisonnable d'envisager un enclos monastique englobant Saint-Vallée et un second dispositif, plus tactique et restreint, ne comprenant que les bâtiments abbatiaux. À l'appui de cette hypothèse, les données archéologiques montrent l'application de cette solution au moins à partir du XIII siècle. Pouvait-elle être antérieure ? La stabilité du tracé des murailles est un élément à prendre en compte quand on s'intéresse au système défensif $d u I^{e}$ siècle: sur le côté oriental $d u$ monastère, elles sont toutes superposées depuis cette période. Étant donné l'influence 
du modèle carolingien dans la mise en place définitive de l'organisation de l'abbaye et les problèmes stratégiques induits par un trop grand périmètre, on est tenté de penser que le tracé médiéval a reproduit celui du IX ${ }^{\mathrm{e}}$ siècle. À défaut de preuve, archéologique ou écrite, cela ne restera qu'une simple hypothèse.

De quelle nature était le rempart? D'une épaisseur moyenne de 2,80 m, il est constitué de deux parements en très gros moellons enfermant un blocage interne. Les extrémités nord et sud n'ayant pas été fouillées, on ne connaît pas son emprise sur la longueur. Il a été en outre très arasé pour l'implantation des fortifications postérieures, refaites aux $\mathrm{X}^{\mathrm{e}}$ et $\mathrm{XIII}^{\mathrm{e}}$ siècles, dont il constitue le soubassement.

35 L'emprise de la muraille carolingienne s'est étendue aux dépens du cimetière préexistant (fig. 10 et 5). Les sépultures qui restaient partiellement visibles ont été réduites de part et d'autre du nouveau mur, mais celles qui étaient entièrement occultées ont été entassées sous forme d'ossuaire sur l'emplacement exact du seuil antérieur. L'enfouissement étant possible ailleurs, cette localisation apparaît volontaire. On est tenté d'y reconnaître une valeur de symbole, en rapport avec la sacralisation de l'ancien seuil, ou plutôt de son emplacement.

Fig. 10 - Emprise de la muraille carolingienne sur le cimetière antérieur : 1) Plan du cimetière ; a) base de la muraille, b) fosses d'inhumations creusées dans le substrat rocheux

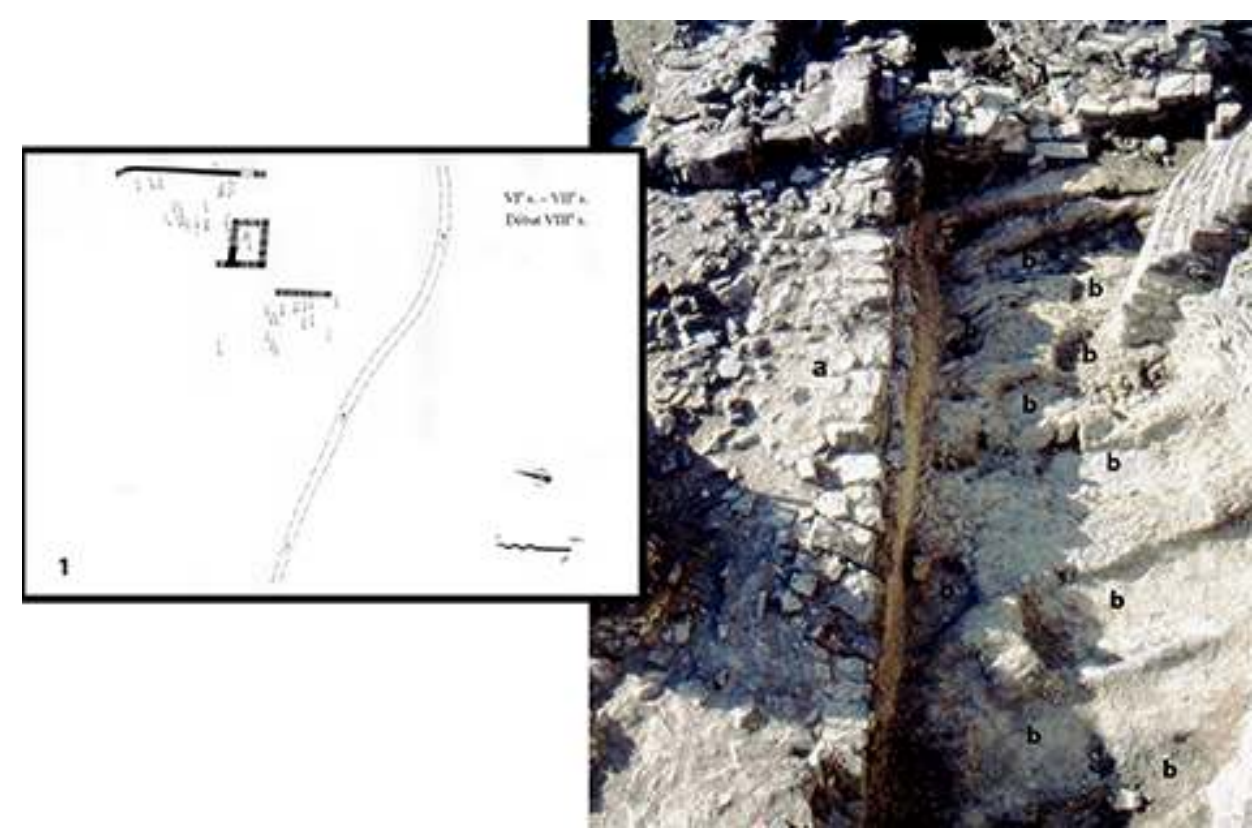

(Cl. des auteurs; DAO V. Bardel)

Une porte, rebouchée par la suite, était ouverte dans la muraille face au chevet plat de l'église, auquel elle était reliée par deux murs perpendiculaires d'environ $1,30 \mathrm{~m}$ de large (fig. 11). 
Fig. 11 - Bases du chevet carré de l'église carolingienne (a : angle nord-est) et des deux murs du porche oriental (b et $\left.b^{\prime}\right)$

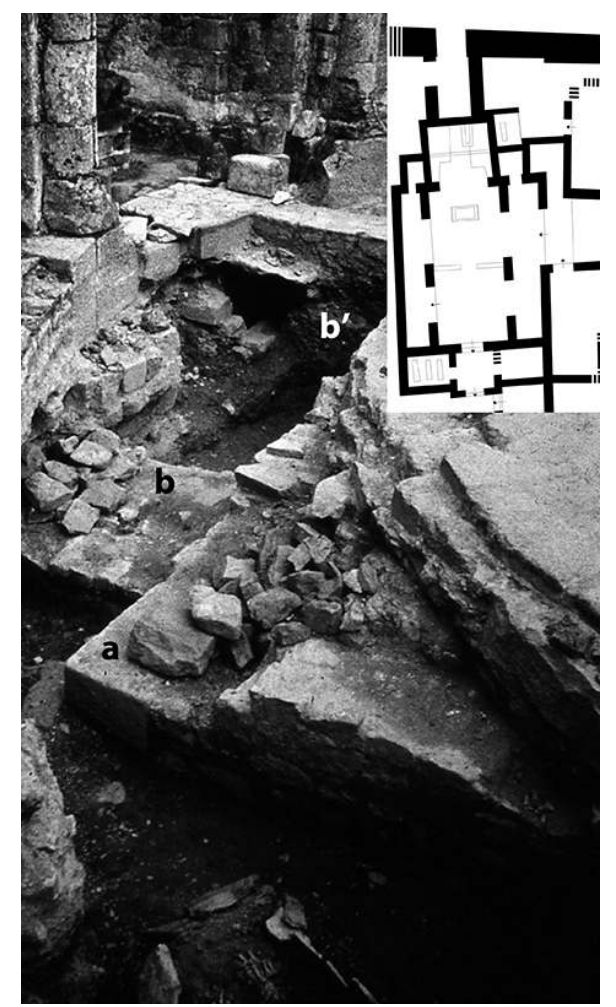

(Cl. des auteurs; DAO V. Bardel)

L'ensemble constitue un porche oriental, ouvert sur son côté nord par un seuil de même largeur que la porte principale, permettant l'accès à l'intérieur de l'enclos. Le cheminement vers l'église nécessitait le contournement du bâtiment par le nord, avant de parvenir à son portail en même temps qu'à une annexe contiguë qui peut représenter la porterie (fig. 8).

Le porche ainsi délimité, intercalé entre la porte du monastère et l'église, remplit une fonction de sas. Il constitue une entrée en chicane permettant de bloquer d'éventuels assaillants comme dans une souricière, après qu'ils ont enfoncé la première porte : le faible battement entre les deux murs ne facilite pas l'usage d'un bélier... Techniquement, la réalisation de l'entrée du monastère traduit l'adaptation à Landévennec de modes plus larges. La porte du castellum de Saint-Denis, dont Charles le Chauve a ordonné la construction en 869 , se trouvait aussi en retrait par rapport à l'enceinte, de manière à créer un " goulot d'étranglement " ${ }^{23}$.

Les études d'élévations et des liants de mortier indiquent que les deux murs gouttereaux du porche sont construits en même temps que l'abbatiale carolingienne, c'est-à-dire dans les débuts du IX $X^{\mathrm{e}}$ siècle. La muraille en est contemporaine, en théorie, à moins qu'elle n'ait été mise en attente. Elle reste cependant antérieure à Gurdisten qui la mentionne vers 870 . Sa datation ne peut être affinée par l'analyse des liants de mortier, puisque les assises basses qui subsistent en sont dénuées. Quoi qu'il en soit, ce n'est qu'après la reconstruction globale du monastère, dans la seconde moitié $d u \mathrm{Ix}^{\mathrm{e}}$ siècle, que les reliques ont été transférées dans le chœur de l'abbatiale (fig. 8-2) ${ }^{24}$.

La fouille n'a pas mis en évidence de fossés antérieurs au XIII ${ }^{\mathrm{e}}$ siècle. La présence d'une douve carolingienne protégeant l'entrée n'est donc pas assurée. Il paraît étonnant que 
les fossés creusés sur les trois autres côtés, mentionnés par Gurdisten, n'aient pas été continués sur la face est, mais il semble que ce soit le cas, à moins que le creusement n'ait été distant de quelques mètres par rapport à la muraille et qu'il ait été de ce fait éclipsé par les douves postérieures.

L'entrée du monastère a donc été déplacée pour être resituée au chevet de la nouvelle église. Le fait en soi n'évoque pas une obligation défensive.

Il faut remarquer que l'aménagement interne du porche oriental laisse un espace libre, une sorte d'impasse à l'arrière du chevet plat. Le fait qu'il se situe derrière le chœur, de l'autre côté du mur contre lequel s'appuie le tombeau de saint Guénolé, évoque aussi un dispositif d'accueil qui aurait permis d'entrevoir la sépulture, par un système d'oculi. Lors des grandes fêtes drainant un nombre considérable de pèlerins, tous les arrivants pouvaient-ils ainsi bénéficier de la vue des reliques? Le niveau d'arasement du chevet ne permet ni de vérifier, ni d'infirmer cette hypothèse ${ }^{25}$.

En résumé, la solution mise en œuvre semble un compromis entre le pratique et le sacré, entre les nécessités stratégiques, l'accueil des pèlerins, etc., et la nécessité de permettre les passages de charrettes. Que l'élaboration de la porte ait été astucieusement réfléchie pour la rendre la plus sûre possible, c'est un fait. Ce qui surprend en revanche, c'est sa localisation. Le chevet de l'église se trouve en effet intégré dans les fortifications abbatiales.

Cette disposition inhabituelle s'explique peut-être par le statut particulier de l'église en tant que lieu de pèlerinage, mais se justifie surtout par la présence des ossements sacrés et la puissance qui leur est accordée. On y a recours lors des sièges pour galvaniser les défenseurs, comme à Paris en $886^{26}$, ou pour redresser une situation perdue. C'est le cas à Tours en 903 : les reliques de saint Martin sont conduites à la porte de la ville déjà presque enfoncée. Leur vue redonne courage aux défenseurs, effraie et met en fuite les Normands, qui sont pourchassés et massacrés ${ }^{27}$.

Il n'est donc pas interdit de penser qu'au dispositif militaire du chevet que constitue l'entrée en chicane, s'ajoute la force des reliques. Celles-ci, partie intégrante du système défensif de l'abbaye, renforcent la protection du lieu le plus vulnérable, la porte. Il faut cependant remarquer que cette localisation particulière de l'entrée face au chevet n'est pas spécifique du monastère carolingien, ni de considérations uniquement stratégiques. Elle était la même en effet aux époques précédentes, lorsque la porte était devant le chevet de l'unique église qui renfermait alors le tombeau de saint Guénolé.

Malgré cette assurance, dans le contexte d'agitation et d'effondrement du pouvoir breton au $\mathrm{x}^{\mathrm{e}}$ siècle, la crainte des Normands devait être telle que les moines préférèrent tout de même fuir avec leurs reliques plutôt que d'affronter les pillards...

Le procédé est toutefois repris à leur retour d'exil, au milieu du $x^{e}$ siècle. Globalement, ils n'entreprennent alors que des travaux de remise en état des bâtiments, mais doivent rebâtir la porte et l'entrée en chicane, mises à bas par les Normands. Cette reconstruction est faite selon le même schéma.

\section{Monachia et claustrum}

Dans le courant $d u$ IX ${ }^{e}$ siècle, la séparation entre espace monastique privé et lieux fréquentés par les laïcs est maintenue sans changement notable. 
La monachia, qui regroupe l'habitat, les lieux de vie, l'église et le cimetière dispose-t-elle d'une clôture spécifique ou celle-ci se confond-elle avec l'enclos monastique, plus étendu a priori ? Hormis la muraille orientale, on ne connaît pas de démarcations sur le pourtour qui pourraient restreindre cet espace en isolant les jardins et lieux cultivés, nécessairement inclus dans le grand enclos. Il est pourtant logique de les imaginer dans la mesure où la monachia apparaît différenciée. La question restera donc en suspens.

En ce qui concerne le claustrum, les limites sont mieux définies. On a mentionné plus haut une base de mur ou de palissade formant à la fois un parvis devant l'oratoire et une barrière au nord de la cour centrale (fig. 7). Elle délimite l'espace purement monastique de l'espace ouvert au public qu'est l'église.

Avec la construction de la basilique, cette disposition est caduque, mais elle est refaite en parallèle. Un solin de pierres, passablement arasé là aussi, forme une clôture nord à partir de la façade de l'église et amorce un retour vers le sud, au bout d'une quinzaine de mètres environ (fig. 8-1). Un mur de soutènement moderne construit sur la suite de cette maçonnerie arasée empêche de la repérer dans son ensemble, mais il est logique de supposer qu'elle rejoint au moins l'aile sud. Le secteur situé plus au sud et comprenant les communs n'ayant pas fait l'objet de fouilles, il est impossible de se prononcer sur la poursuite de cette clôture. On constate qu'elle est conservée telle quelle lors de la reconstruction du monastère au $x^{e}$ siècle (fig. 8-2). Mais le simple fait que la nouvelle ordonnance du bâti soit orthonormée délimite une cour fermée, nettement individualisée, qui n'existait pas auparavant. Les bâtiments s'agençaient bien autour d'une cour qui fonctionnait comme le centre de la circulation interne et de l'organisation du monastère. Mais elle n'apparaissait fermée que sur ses faces nord et ouest. Cela peut être dû au fait que les fouilles sont incomplètes au sud et que, si clôtures il y avait, on ne les connait pas. Il est cependant indubitable qu'en étant placée à la perpendiculaire de l'aile orientale, l'aile sud achève de transformer cette cour en claustrum.

Cet effet est renforcé par une galerie couverte, soutenue par une colonnade, qui dessert les deux ailes, au sud et à l'est, sur toute leur longueur. La circulation interne apparaît ainsi codifiée, bien que la fonction des divers bâtiments ne change pas. L'aile sud reste dévolue au réfectoire ainsi qu'aux cuisines et celliers, et l'aile orientale concentre les lieux réguliers proprement dits. La salle centrale bénéficie alors d'un traitement particulier, non identifié jusqu'à présent : large baie côté est, porte rehaussée de deux piliers destinés à recevoir les retombées des arcades de la galerie. Cette salle est restée la salle du chapitre du monastère jusqu'à la Révolution.

51 La cour autour de laquelle s'ordonnancent l'église, le chapitre, le réfectoire et la cuisine, avec sa colonnade sur deux côtés, évoque fortement le cloître tel qu'on le connaîtra par la suite. Mais son ampleur et sa disposition les rapprochent aussi des cours de villae gallo-romaines. En effet, une porte cochère ouverte dans le mur qui la limite au nord, non loin de l'église, oblige à y envisager des passages charretiers vers l'extrémité de l'aile sud (le cellier ?). Cette circulation est limitée à la partie haute de la cour et la colonnade est interrompue avant son extrémité. Aucune démarcation n'a été mise au jour entre cette zone de passage et le reste de la cour. Si cela est avéré, cette mixité paraitt incompatible avec le caractère d'un cloître.

Le claustrum carolingien apparaît donc comme les prémices d'un cloître, mais l'évolution vers le cloître classique ne s'est faite que progressivement. 


\section{Un chancel}

52 Landévennec étant une église de pèlerinage, la circulation devait y être nécessairement très codifiée. On ne possède pas d'éléments à ce sujet, qu'ils soient écrits ou archéologiques, hormis pour ce qui concerne l'existence d'un chancel. Son établissement répond au souci de satisfaire aux exigences canoniques: l'église est accessible aux laïcs, mais cet accès est limité. Le concile de Vannes de 658 rappelait l'obligation d'établir une barrière et de ne pas admettre les femmes au-delà de celle$\mathrm{ci}^{28}$. Dans la Vita de saint Guénolé, Gurdisten nous apprend que les femmes n'ont plus accès au chœur de l'église, depuis une intervention vigoureuse de l'épouse de Pétran pour y chercher son mari... ${ }^{29}$.

53 À l'époque carolingienne, la nef de la grande église était séparée de l'avant-chœur par une barrière dont on ne connaît ni l'aspect ni la composition. Les fouilles ont permis de mettre au jour la base arasée d'un mur étroit, engagé sous les deux piles romanes de la croisée du transept et qui comporte une ouverture centrale de 1,10 m de large (fig. 8). Aucun élément architectural n'a été retrouvé en dehors de cette maçonnerie, qui soutenait probablement un chancel. Elle est absente des bas-côtés, ce qui laisse envisager l'existence de deux passages latéraux. Un dispositif semblable est par exemple décrit dans la Vita de sainte Brigitte ( vII $^{\mathrm{e}}$ siècle) ${ }^{30}$, qui figurait d'ailleurs en bonne place dans la bibliothèque de l'abbaye ${ }^{31}$.

Le cloisonnement de l'espace semble renforcé par le traitement des surfaces. Les différentes couleurs de mortier de sol utilisées dans l'église matérialisaient les zones autorisées ou interdites aux fidèles : couleur ocre dans l'avant-chœur, blanche dans la nef. Le chancel carolingien a été conservé, semble-t-il, sous sa forme originelle au $\mathrm{x}^{\mathrm{e}}$ siècle et dans le premier tiers $\mathrm{du} \mathrm{XI}^{\mathrm{e}}$ siècle, et la différenciation des sols jouait seulement sur les matériaux : un sol de tuileau dans la nef et un carrelage de terre cuite dans l'avant-chœur.

\section{Une absence de clôtures à l'époque romane?}

Des traces de réfection postérieures attestent la survivance d'une barrière de chœur au Moyen Âge. D'après les données archéologiques, elle devait être en bois au $\mathrm{XI}^{\mathrm{e}}$ siècle, avec deux poteaux délimitant la porte centrale. L'absence de restes ne permet pas de savoir si elle était plus proche du chancel que du jubé.

Paradoxalement, la seule limite connue pour l'époque romane est celle du chœur de l'église. Si l'on excepte les aménagements du cloître, les autres démarcations nous sont inconnues. La muraille orientale, reconstruite ou restaurée au $\mathrm{x}^{\mathrm{e}}$ siècle, est alors épierrée et rasée et aucun vestige n'a été retrouvé de la clôture qui l'a très probablement remplacée. Était-elle plus proche de la mer, comme cela s'est produit par la suite?

57 L'enceinte a peut-être été agrandie, mais l'un des principaux éléments à prendre en compte vient du fait que l'entrée de l'abbaye a été, dès cette période, transférée de l'est au nord-ouest, du côté où se situe l'accès actuel au site. Le monastère regarde à présent non plus vers la mer, mais vers le bourg. La construction du bras nord du transept et le rallongement de l'église par rapport à la précédente ont condamné l'ancien chemin d'arrivée au monastère. Mais ce n'est probablement pas la cause de ce changement. Il 
faut considérer que c'est vers cette époque que Landévennec s'est vue ériger en paroisse autonome, ou tout au moins en trève d'Argol ${ }^{32}$. L'église de la communauté villageoise est alors à la nomination de l'abbé de Landévennec, comme c'était aussi le cas d'Argol. Après sa fondation, l'église paroissiale et le cimetière qui l'entourait ont été implantés à proximité immédiate de l'abbatiale et de la nouvelle porte ${ }^{33}$ (fig. 12). Étaient-ils inclus dans l'enceinte de l'abbaye, comme cela est assuré à l'époque moderne ? Et si oui, comment la limite entre cimetières paroissial et abbatial était-elle matérialisée?

Fig. 12 - Localisation sur fond cadastral de la paroisse primitive : (a) entrée de l'abbaye moderne : (b) emplacement de l'église paroissiale et du cimetière environnant (parcelles 1170-1171-1174)

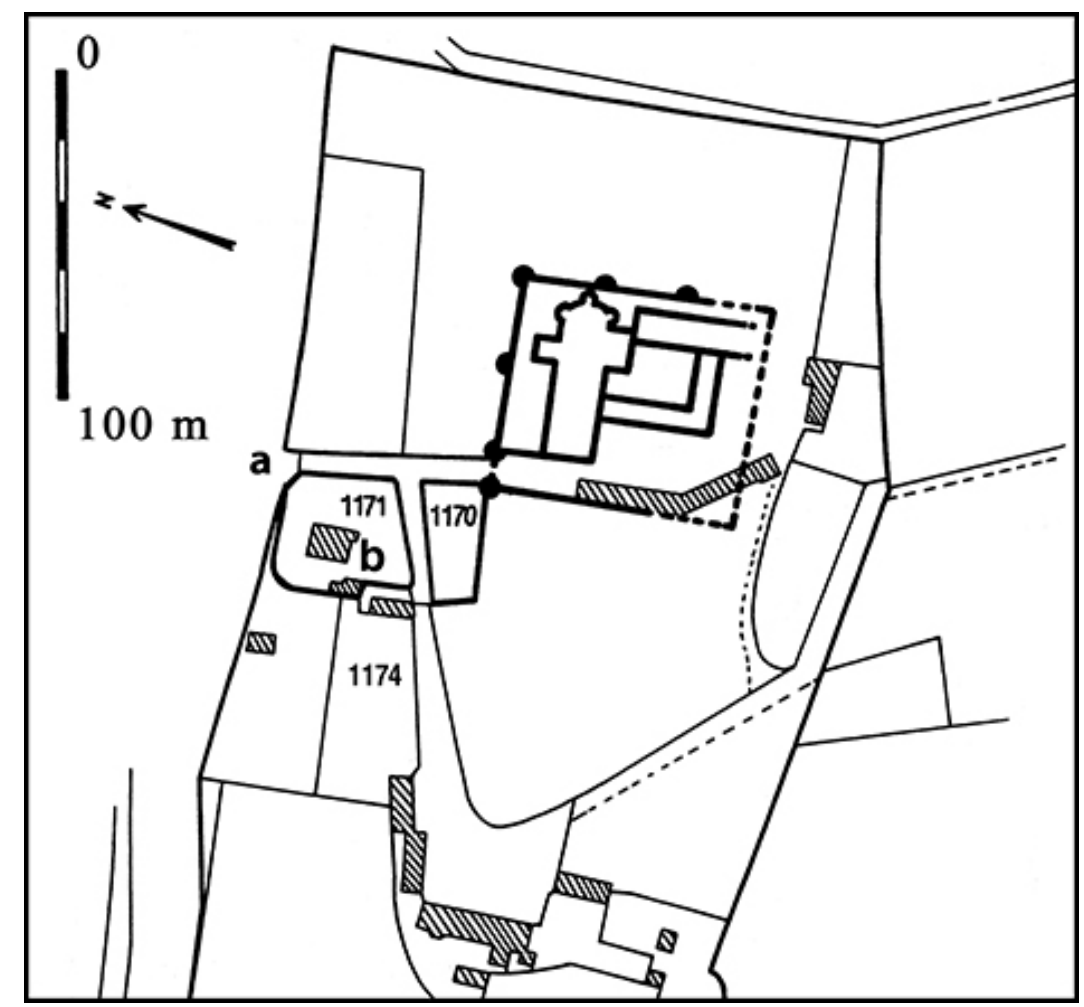

(DAO V. Bardel)

Ou bien restaient-ils à l'extérieur de la clôture ? Il n'est pas impossible en effet que le bourg monastique ait été préalablement installé contre les murs, selon une organisation somme toute comparable à celle de la basse-cour d'une motte féodale. Étant donné la localisation de l'église paroissiale, cela impliquerait que l'enceinte du XI siècle était plus resserrée que celle du bas Moyen Âge ou de l'époque moderne, ou même que l'enclos monastique carolingien.

L'absence de données archéologiques ne permet pas, en l'état des connaissances, de vérifier cette hypothèse. Il faut toutefois signaler la découverte de traces d'occupation médiévale entrevues lors du creusement d'une tranchée de drainage, au nord de l'église. L'emplacement de ces vestiges correspond au pied du rempart nord de la fortification $\mathrm{du} \mathrm{XIII}^{\mathrm{e}}$ siècle. De là à en déduire qu'il s'agissait déjà de la limite de l'enclos monastique $\mathrm{au} \mathrm{XI}^{\mathrm{e}}$ siècle... Ces indices sont beaucoup trop fugaces pour étayer une théorie. 
60 Ce déplacement de l'est vers le nord-ouest a certainement été facilité par une longue période de tranquillité qui justifiait l'absence de fortifications constatée aux $\mathrm{XI}^{\mathrm{e}}$ et $\mathrm{XII}^{\mathrm{e}}$ siècles. Mais cela n'excluait pas les clôtures. Il est probable que l'extension de l'enclos monastique, en lien avec les changements de statuts religieux, a reporté les bordures au-delà des zones fouillées.

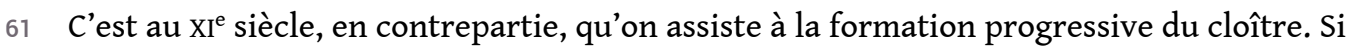
celui-ci émerge avec le monastère carolingien, il continue son évolution avec la construction de l'église romane.

Dans les années 1020-1030, il diffère peu du claustrum carolingien. C'est une très grande cour qui occupe tout l'espace compris entre les deux bâtiments monastiques et l'église romane en construction (fig. 13). Celle-ci ayant été considérablement rallongée, puisqu'elle double en longueur la basilique carolingienne, elle ferme naturellement la cour au nord. Elle recouvre ainsi le mur de clôture du IX siècle qui est rasé sur toute sa longueur, mais on ne sait pas ce qui le relaie côté ouest. Des portiques soutenus par des poteaux de bois remplacent la colonnade carolingienne qui disparaît progressivement et une galerie supplémentaire assure maintenant la desserte du secteur nord.

Fig. 13 - L'église romane en cours de construction englobe la basilique carolingienne. La circulation dans la cour est complétée par des portiques en bois

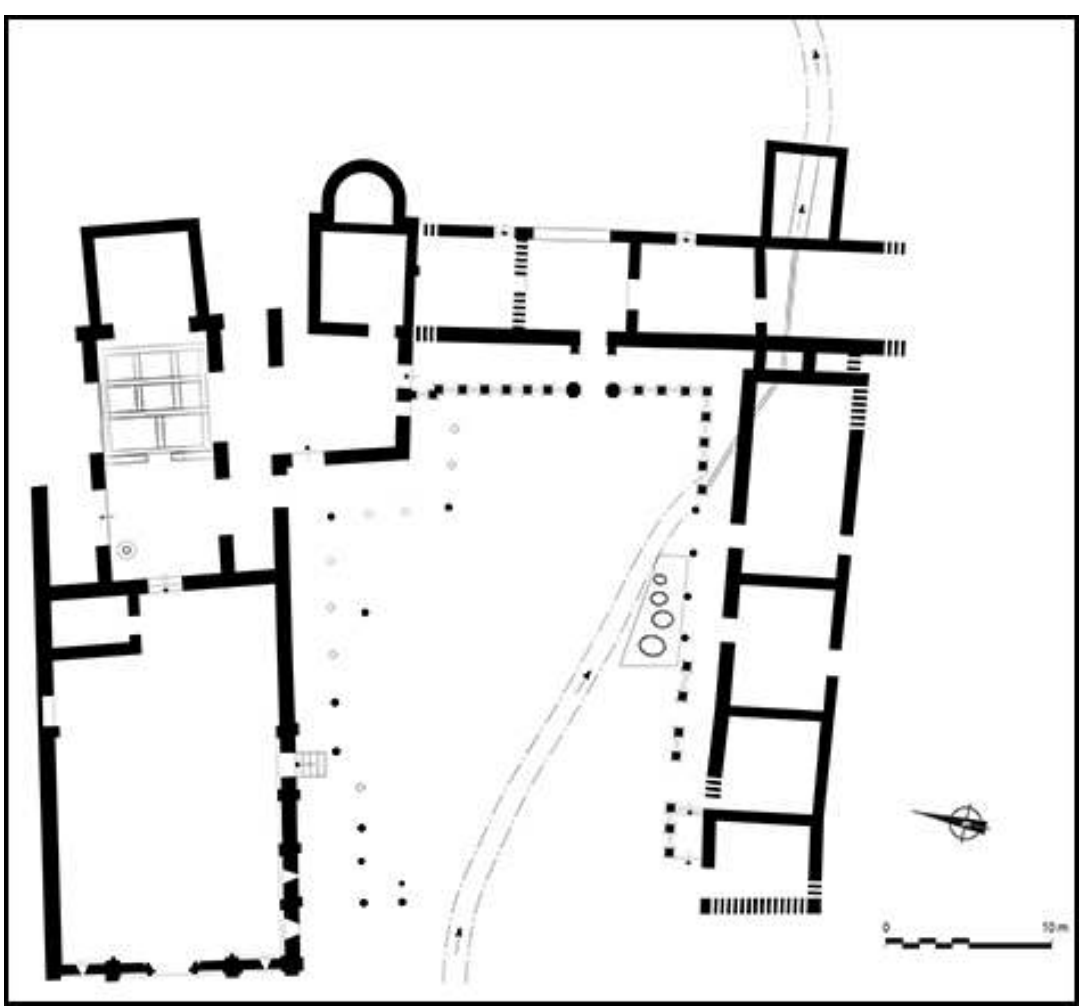

(DAO V. Bardel)

Dans l'angle nord-ouest, les poteaux forment un retour qui évoque la présence d'un mur contre lequel ils pourraient logiquement s'appuyer et qui assurerait la clôture occidentale. L'impossibilité de fouiller cette bande de terrain a empêché de le mettre en évidence.

Comme le claustrum du IX ${ }^{\mathrm{e}}$ siècle, celui-ci n'est pas quadrangulaire puisque les galeries suivent les murs de l'église côté nord. 
63 vers 1050, lorsqu'une aile occidentale est construite en travers de la cour (fig. 14). Celleci est alors divisée entre un dispositif claustral et une aire probablement close, mais ouverte à la circulation fonctionnelle du monastère. Ainsi les déplacements entre église, chapitre et réfectoire sont dissociés des passages charretiers traversant la cour pour mener aux communs.

\section{l'aile occidentale. Mais le cloître n'est toujours pas quadrangulaire.}

Les quatre galeries du cloître classique n'ont été mises en place qu'au XIII ${ }^{\mathrm{e}}$ siècle.

Fig. 14 - Le monastère roman au milieu du $x \underline{e}$ siècle

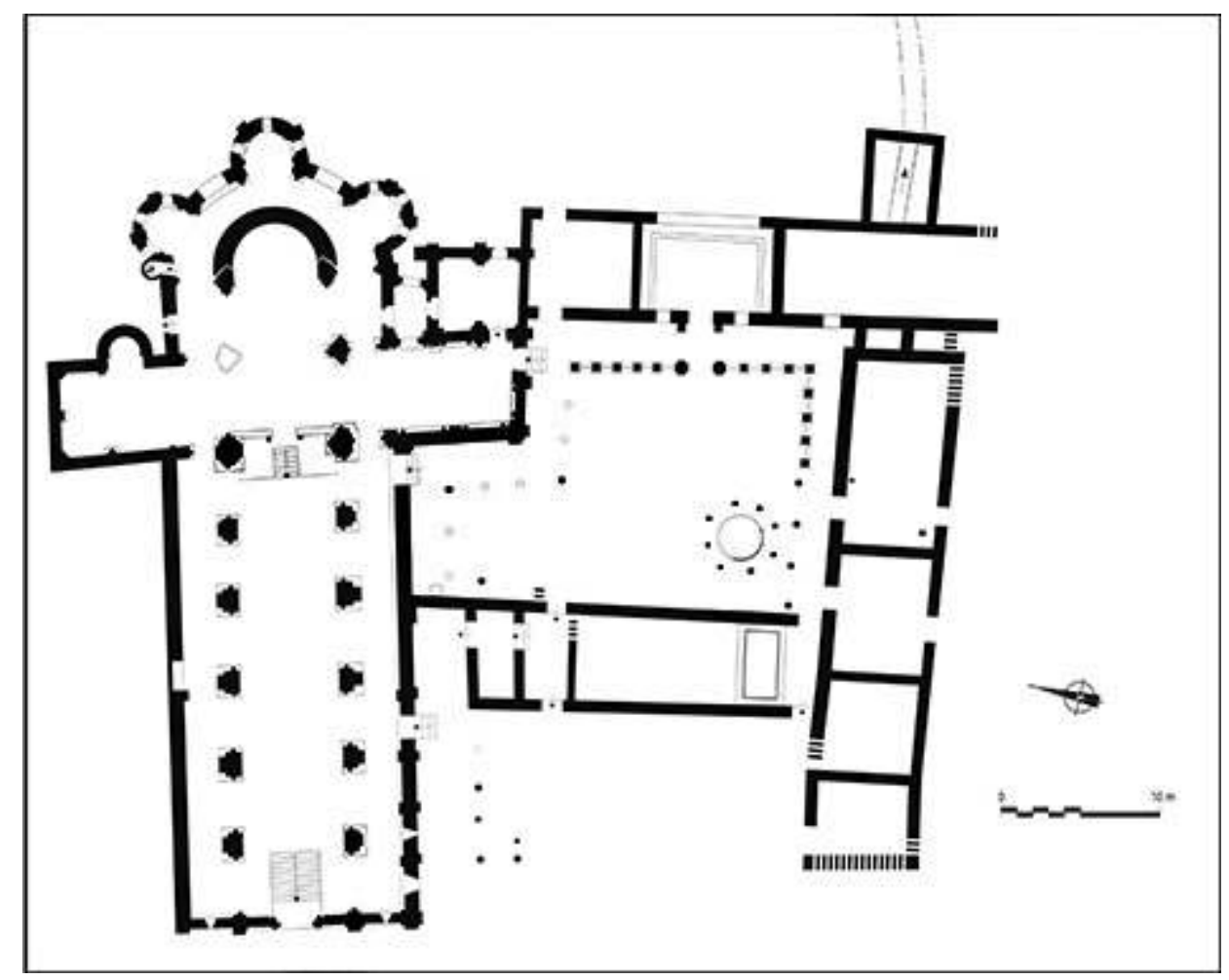

(DAO V. Bardel)

\section{Un surcroît de clôtures au XIII ${ }^{\mathrm{e}}$ siècle?}

Plutôt que d'un surcroît, on pourrait parler d'une extrême concentration des clôtures pour cette période. Encore faut-il préciser que c'est le castrum qui est devenu envahissant : il se confond avec la monachia, qui n'est plus différenciée.

Il existe nécessairement un enclos monastique plus large, mais qui peut être le même qu'au $\mathrm{XI}^{\mathrm{e}}$ siècle. $\mathrm{Et}$ comme pour le $\mathrm{xl}^{\mathrm{e}}$ siècle, il manque les éléments nécessaires à sa restitution. Il est donc impossible de savoir de quelle façon l'ensemble des clôtures périphériques a évolué depuis l'époque carolingienne.

67 Au milieu du xiII siècle, sous la menace des raids anglais, le rempart oriental est reconstruit sur les bases que constituent les anciennes fortifications arasées. Ce qui en a été mis au jour est constitué d'un mur de moellons large d'1,50 m, renforcé de

Bulletin du centre d'études médiévales d'Auxerre | BUCEMA, Hors-série n 12 | 2020 
contreforts internes massifs et réguliers, qui supportaient probablement un chemin de ronde en bois. La muraille était épaulée de tours semi-circulaires de 5,50 $\mathrm{m}$ de diamètre en moyenne, qui ont pu être localisées en partie (fig. 15) ${ }^{34}$. Les bases de deux d'entre elles ont été dégagées au cours des fouilles opérées sur la face orientale des fortifications.

Fig. 15 - Le monastère au milieu du XIII

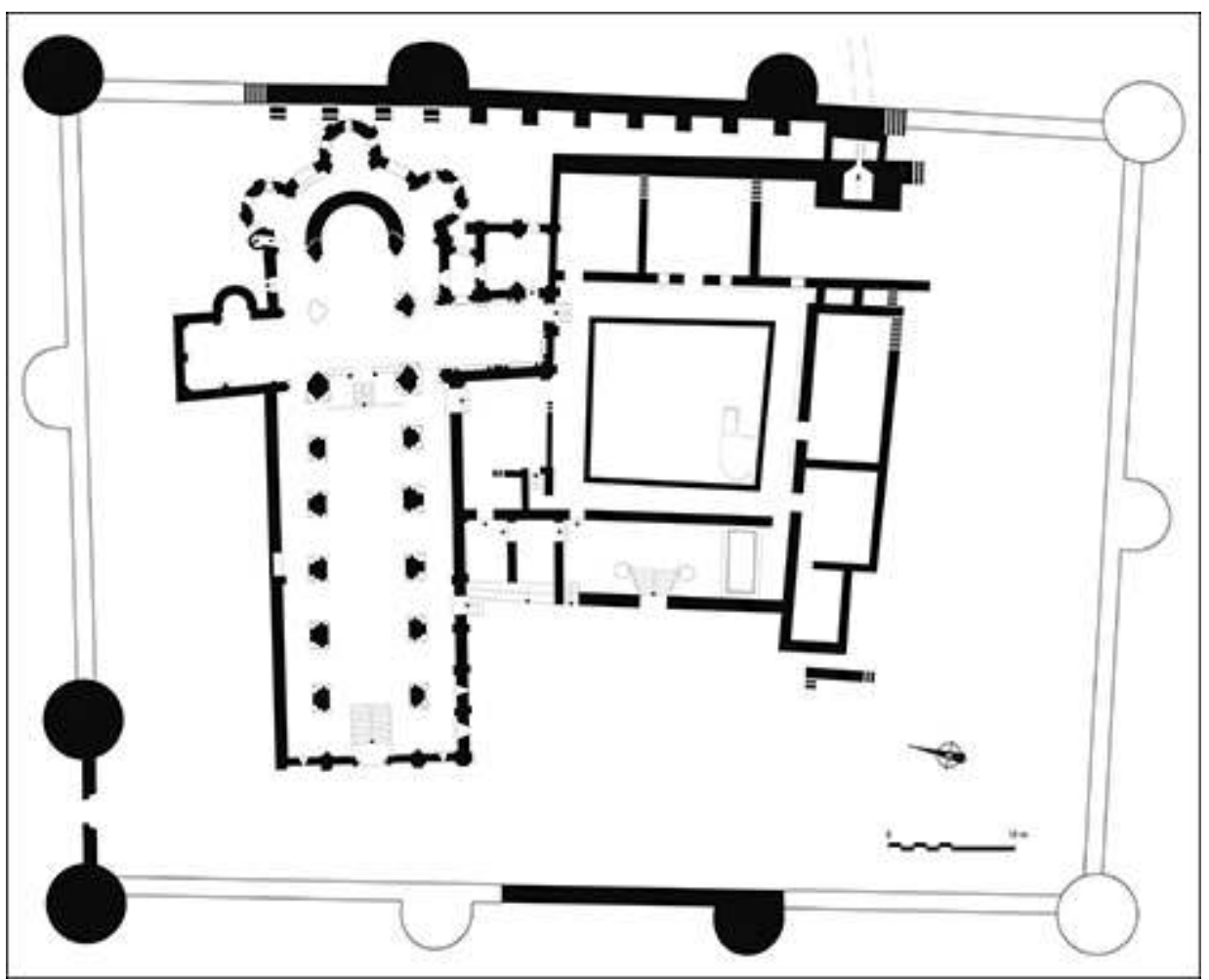

(DAO V. Bardel)

Deux autres ont été repérées grâce à des clichés aériens, aux deux extrémités de la face nord. On sait par les plans du xvII ${ }^{\mathrm{e}}$ siècle que celle de l'extrémité ouest était jumelée avec une tour conservée en élévation et qu'elles constituaient auparavant un châtelet d'entrée (fig. 16) ${ }^{35}$. Ce dispositif était situé juste dans l'angle nord-ouest et il se raccordait à un mur de clôture moderne, visible également sur le plan, qui occupe l'emplacement de la courtine arrière. En effet, lors des travaux de terrassement préliminaires à la construction du musée, les restes d'un pan de muraille assorti d'une tour ont été provisoirement dégagés, côté ouest. Cela a permis de localiser le rempart arrière et d'y confirmer l'existence des mêmes tours semi-circulaires, disposées en symétrie par rapport à la face orientale.

Des douves renforçaient ces remparts. Une partie en a été partiellement fouillée du côté de la mer, mais comme elles ont été recreusées au $\mathrm{Xv}^{\mathrm{e}}$ siècle, les dimensions dont on dispose sont celles des plus récentes (10 $\mathrm{m}$ de largeur pour $3 \mathrm{~m}$ de profondeur). Comme pour le reste des fortifications, les terrassements préliminaires au musée nous ont permis de localiser et relever la suite des douves en plusieurs emplacements, sur les faces ouest et sud. Il semble qu'elles avaient sensiblement les mêmes dimensions, peutêtre un peu plus réduites sur le côté sud, très en pente vers la mer et où les affleurements rocheux étaient superficiels. Vu l'emplacement de ce fossé sud, il est 
logique de projeter un quatrième côté aux fortifications (fig. 15). Il se situe tout d'abord dans l'alignement d'une quatrième tour orientale, une tour d'angle qui serait implantée à distance égale des trois autres, ce qui apporte une certaine crédibilité au tracé proposé. Mais il faut considérer en outre que ce côté du monastère est au bas d'une colline très pentue, facilement accessible par la grève, qui serait une rampe d'accès particulièrement favorable à des attaquants si elle n'était barrée par un fossé et un rempart. De plus, cette disposition laisse une place pour les communs entre l'aile sud et la fortification.

Le château fort conçu par les moines, car c'est bien de cela dont il s'agit, enserrait au plus près les bâtiments, afin d'en permettre la défense. L'abbaye vivait, au propre comme au figuré, à l'ombre de ses murs. Cette disposition avait au moins le mérite de l'efficacité : une enquête du vicomte d'Avranches signale, en 1296, deux raids anglais sur l'abbaye se soldant à chaque fois par un échec. Demi-échec serait plus approprié, car si “ceus qui estoient dedenz en la compagnie de l'abbé se défendirent en tel manière que eus ne meffirent onques à l'abbaïe", la fortification n'empêche pas les assaillants de brûler la ville et deux grands bateaux et d'emporter du blé, du sel, du "boiz à fere vessiaus" ${ }^{36}$.

71 Tout cela n'interrompt pas l'accès à l'église. Il se fait par la porte du château, qui communique avec le bourg. S'il y a eu de nouveaux aménagements du lieu de culte, ils ne sont pas visibles dans les bases conservées actuellement. Seule la barrière de chœur garde les traces d'une modification : des dés maçonnés remplacent les poteaux de bois de part et d'autre de l'ouverture, mais sur le même emplacement, c'est-à-dire accolés à la murette. Ces bases soutiennent une structure dont l'élévation et la composition sont inconnues.

Quant au cloître, qui se retrouve au centre du système, il connaît à ce moment son aboutissement. Avec ses quatre galeries disposées en carré et ses colonnettes doubles posées sur un mur-bahut, il correspond à l'image-type du cloître médiéval.

Il faut noter cependant que la galerie orientale étant dans l'alignement du transept sud depuis l'époque carolingienne, il se posait le problème de la jonction avec l'église. En effet, la cour ne peut s'appuyer contre la nef, contrairement à l'usage courant. Cela a été résolu par la transformation de l'espace situé sous le transept en salle annexe dont on ne connait d'ailleurs pas la destination. Le préau peut ainsi s'inscrire dans un quadrilatère (fig. 15). Contrairement à l'époque romane, où les colonnes de bois faisaient toute la hauteur du portique, un mur-bahut délimite et isole les galeries. Les colonnettes et leurs chapiteaux, ainsi que les dalles de couronnement du mur, sont alors taillés dans une pierre calcaire importée de $\mathrm{Caen}^{37}$. On peut imaginer l'effet ostentatoire de ce cloître blanc inséré au cœur du château fort qu'est devenu le monastère.

\section{En conclusion}

L'emprise de la clôture est d'autant plus difficile à restituer qu'elle s'est souvent confondue, au moins partiellement, avec les dispositifs de protection de l'habitat monastique. Quant aux clôtures périphériques, on en connaît surtout l'état actuel, c'est-à-dire celui de l'époque moderne. 

Plouvier ainsi que de la gravure du Monasticon Gallicanum $^{38}$, que ce soit pour le monastère bâti ou pour son environnement immédiat. Ils révèlent une multiplicité des niveaux de clôture qui posent question. Sont-ils représentatifs de ce qui existait précédemment dans les faits ou simplement le résultat d'une accumulation progressive?

En ce qui concerne la monachia, le cloître reste une cour à quatre galeries entièrement incluses dans les bâtiments monastiques (fig. 16). À l'intérieur de l'église, comme dans toutes les abbatiales, on sait qu'une grille de bois de chêne maintenait encore, à la fin du XVIII ${ }^{\mathrm{e}}$ siècle, la séparation entre nef et chœur ${ }^{39}$.

Fig. 16 - Plan d'ensemble des structures fouillées avec l'environnement du XVII plan du premier étage de R. Plouvier)

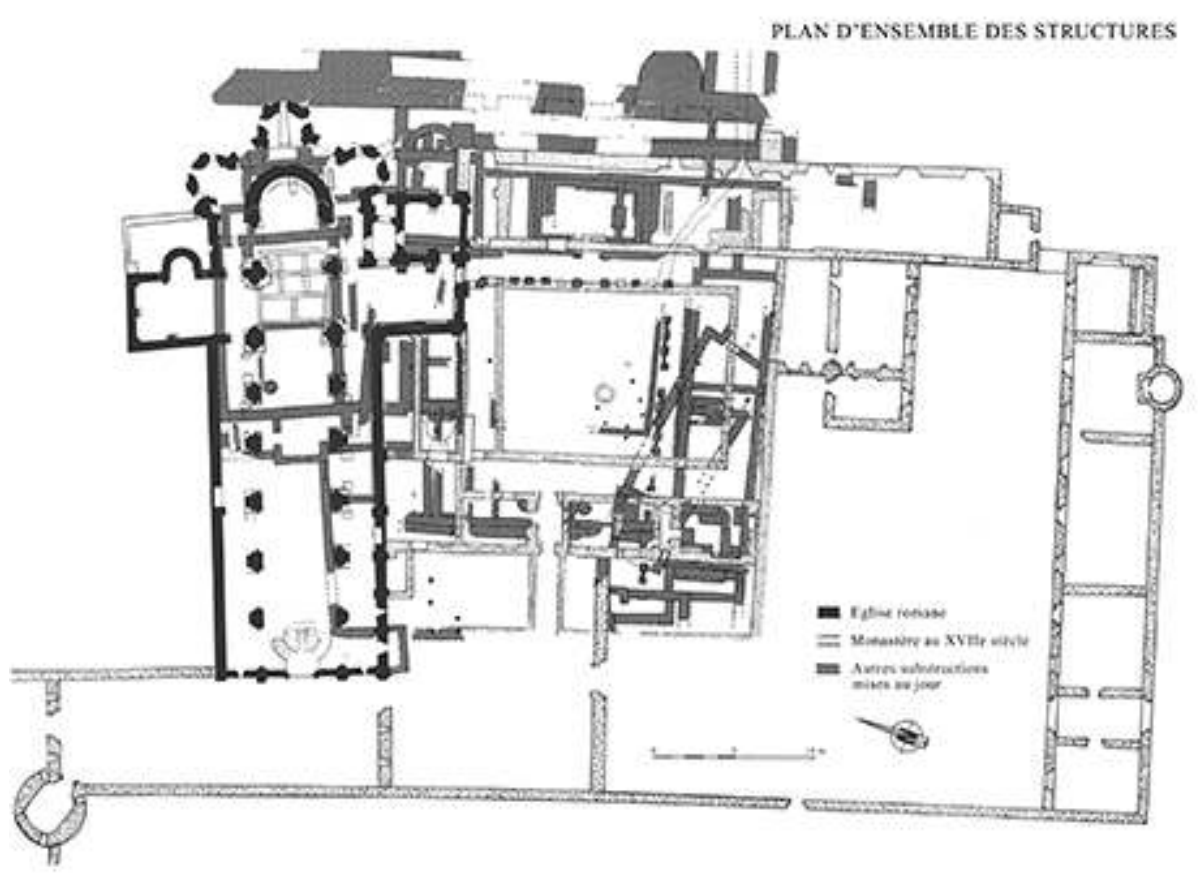

$\underline{(D A O ~ V . ~ B a r d e l) ~}$

Mais à l'extérieur, une première barrière ferme l'accès au parvis. À sa limite sud, un deuxième mur clôt le passage vers l'accueil du monastère, c'est-à-dire l'aile ouest, à travers une cour intérieure qui était apparue dès le $\mathrm{XI}^{\mathrm{e}}$ siècle, avec la mise en place de cette aile occidentale. On ne connaît pas la fermeture de cette cour à l'époque romane. Elle se fait ici par un mur ouest qui assure la clôture de ce côté depuis l'entrée jusqu'audelà des communs et qui sépare la monachia de l'enclos monastique.

Dans l'espace de circulation que délimite ce mur et qui dessert l'église et le monastère, une troisième barrière donne accès aux communs.

Si l'on en croit les canonnières nettement visibles sur le plan, et qui n'ont guère de raison d'avoir été créées au milieu du $\mathrm{xVII}^{\mathrm{e}}$ siècle, cette triple démarcation date probablement des $\mathrm{Xv}^{\mathrm{e}}$ ou $\mathrm{XvI}^{\mathrm{e}}$ siècles. En effet, après les guerres de succession de Bretagne, le monastère a été en grande partie reconstruit et le système défensif restauré : réfection des douves, aménagements d'artillerie. 
79 Elles ne sont pas remplacées, mais un mur de clôture élargi délimite dès lors l'ensemble du domaine, du côté de la mer, qu'il côtoie, et du bourg, où il inclut l'enclos paroissial jusqu'au milieu du siècle, repoussant vers le nord la porte du monastère. Entre cette porte et celle de l'accès au parvis de l'église, une voie canalisée est instaurée pour les laïcs, bordée du côté oriental par un mur isolant le verger et du côté ouest par une autre clôture, inexistante avant le XVII siècle, qui ferme le domaine privé de l'abbé.

Loin de se restreindre, les délimitations semblent donc s'exacerber à l'époque moderne, comme en témoigne le Mémorial de Landévennec qui indique qu'en 1650, «la partie du mont dit Penforn qui surplombe le monastère du côté de l'occident fut jointe à la clôture par un fossé large et profond tracé et dégagé tout autour d'elle $»^{40}$.

\section{NOTES}

1. B. TANGUY, A. DIZERBo, M. SIMON osb, «Les possessions de l'abbaye ", in M. SIMON osb, L'abbaye de Landévennec, de saint Guénolé à nos jours, Rennes, 1985, p. 184-195. W. DAVIES, «Les chartes du Cartulaire de Landévennec", in Landévennec et le monachisme breton dans le haut Moyen Âge, Bannalec, 1986, p. 85-95. B. TANGUY, «Les noms de lieu dans le cartulaire de Landévennec », in Landévennec et le monachisme breton, op. cit., p. 141-153.

2. C. de SMEDT, "Vita sancti Uuinuualoei abbatis Landevenecensis, auctore Wurdestino, nunc primum integre edita », Annalecta Bollandiana, t. VII, 1888, p. 167-261. Il s'agit de la Vita Amplior Winwaloei à laquelle on se référera sous une forme abrégée : VAW [livre] I ou II, [chap.] 7, etc.

3. Au IX ${ }^{\mathrm{e}}$ siècle, dans la description que Gurdisten fait du monastère tel qu'il devait être à l'arrivée de Guénolé, la part de la forêt est très importante: le lieu est en effet présenté comme une clairière.

4. Les bases de la datation sont issues d'analyses radiocarbone sur l'un des squelettes les plus anciens du cimetière: Ly-5783, dates probables 384 à 618, et sur un sol de circulation contemporain : Ly-14128, dates 423 à 571 . C'est par relations stratigraphiques que ces éléments ont été liés entre eux ainsi qu'avec l'oratoire, et les fourchettes obtenues ont été affinées, en particulier grâce à une étude de comput pascal qui ne rendait possibles que deux dates : fin $\mathrm{V}^{\mathrm{e}} \mathrm{s}$. ou fin VII $^{\mathrm{e}}$ s.: B. MERDRIGNAC, "L'âge des Saints", in Études celtiques, t. XXXIV, (1998-2000), p. 161-184. Toutes les datations avancées dans cet article sont issues essentiellement des données stratigraphiques, systématiquement recoupées avec les autres éléments chronologiques qui peuvent être pris en compte.

5. A. BARDEL, R. PÉRENNEC, «Le monastère de Landévennec au temps du cartulaire ", in S. LEBECQ (dir.), Cartulaire de Saint-Guénolé de Landévennec, Rennes, 2015, p. 77.

6. C. de SMEDT, VAW II, 5, p. 219.

7. A. BARDEL, "Les premiers bretons à Landévennec ", in P.-R. GIOT, B. MERDRIGNAC, P. GUIGON Les premiers bretons d'Armorique, Rennes, 2003, p. 129.

8. P. ARIÈs, L'Homme devant la mort, t. 1, Le temps des gisants, Paris, 1985, p. 44 (Points Histoire).

9. Datations antérieures au milieu du $\mathrm{VII}^{\mathrm{e}}$ siècle d'après les données stratigraphiques. Recoupement avec des analyses ${ }^{14} \mathrm{C}$ sur des sols charbonneux : Ly-5781 (473 à 657) et Ly-14125 (555 à 657), avec des pics de probabilités autour de 610 dans les deux cas.

Bulletin du centre d'études médiévales d'Auxerre | BUCEMA, Hors-série n 12 | 2020 
10. P. ARIÈs, op. cit., p. 44.

11. C. de SMEDT, op. cit., 7, p. 221. Le terme employé par Gurdisten est « claustrum », qui a chez lui une signification assez large et variée.

12. C. de SMEDT, op. cit, 23, p. 236.

13. Deux analyses radiocarbone sur des niveaux contemporains du mur (Ly-12567 et 12570) ont fourni des datations très proches avec des pics entre 605 et 658.

14. Pratique courante en Irlande selon J.-M. PICARD, communication personnelle.

15. A. BARDEL, R. PÉRENNEC, "Les monastères précarolingiens de Landévennec ", in Britannia Monastica, 20 (2018), à paraître.

16. Les niveaux de sols de même contexte stratigraphique, sur tout l'ensemble de la cour centrale, étaient très nettement ancrés dans le milieu du VII ${ }^{\mathrm{e}}$ siècle, grâce à plusieurs analyses radiocarbone Ly-9174, 9176, 9177, 14121, 14122, 14123, qui présentent chacune un seul pic de probabilités, les six étant répartis entre 653 et 668 . Cela a permis d'établir un étalonnage dendrochronologique dans lequel s'inscrit le poteau le mieux conservé, avec une date d'abattage en 662 : Y. LE DIGOL, Y. COUTURIER, V. BERNARD, Abbaye Saint-Guénolé, Landévennec: rapport d'étude dendrochronologique, Dendrotech, CNRS, Rennes, 2008.

17. Cette clôture n'a pu être datée que par relations stratigraphiques.

18. La datation de l'église s'appuie sur deux analyses ${ }^{14} \mathrm{C}$ (foyers contemporains de sa construction) : Gif-7124 et Gif-6766 dont les fourchettes assez larges (693 à 986) ont pu être affinées du fait d'impossibilités stratigraphiques et par recoupement avec les datations dendrochronologiques de deux cercueils qui seraient isolés s'ils n'étaient fixés par le seuil de l'église. Ils ont le même terme (780), mais l'absence d'aubier les situe dans les années postérieures (Y. LE DIGOL et al., op. cit.).

19. M. SIMON osb, L'abbaye de Landévennec, op. cit., p. 50, 282. Wallois, Valloé ou Vallée dérivent de Winwalloei qui a donné par la suite Guénolé, attesté à la fin du XI ${ }^{\mathrm{e}}$ siècle.

20. La tradition des chapelles multiples était bien ancrée dans les monastères à l'époque carolingienne ( $c f$. "Chapelles et oratoires monastiques », Table ronde, $5^{\mathrm{e}}$ rencontre des Ateliers clunisiens, CEM et ENSAM, Cluny, sept. 1997). Il est étonnant cependant que Gurdisten ne mentionne jamais cette chapelle.

21. C. de SMEDT, op. cit., 5, p. 219.

22. Le secteur oriental a fait l'objet de fouilles programmées pendant plusieurs années, entre 1985 et 1990 , puis en 1999-2000.

23. C. HERON, O. MEYER, M. WYSS, "L'environnement urbain du monastère de Saint-Denis ", in J. CUISENIER, R. GUADAGNIN (dir.), Un village au temps de Charlemagne, Paris, 1988, p. 67.

24. Constat établi d'après les relations stratigraphiques.

25. Ce système d'oculi au chevet a été évoqué aussi par Uwe LoBBEDEY lors d'une visite sur le site (communication orale)

26. A. D'HAENENS : Les invasions normandes, une catastrophe?, Paris 1970, p. 59-60.

27. Ibid.

28. G. BERNIER, Les chrétientés continentales depuis les origines jusqu'au IXe siècle, Saint-Malo, 1982, p. 186 (Dossiers du Ce.R.A.A, 10).

29. J.-C. CASSARD, Les Bretons de Nominoë, Brasparts, 1990, p. 251.

30. Vita de sainte Brigitte par Cogitosus, in G. BERNIER, op. cit.

31. J.-L. DEUfFIC, « Les manuscrits de Landévennec », in M. SIMON, op. cit., p. 261.

32. R. PÉRENNEC, A. BARDEL, «La paroisse à Landévennec, émergence et évolution », in J.-C. CASSARD et al. (dir.), Mélanges offerts au professeur Bernard Merdrignac, in Britannia Monastica, 17 (2013), p. 250-252.

33. L'église paroissiale n'a été transférée à son emplacement actuel, sur le bord de la mer, à l'autre extrémité du bourg, qu'au milieu du XVII ${ }^{\mathrm{e}}$ siècle. 
34. Les contreforts de pierres, d'environ $1,25 \mathrm{~m}$ au carré, étaient espacés de $2,80 \mathrm{~m}$. On ne sait pas s'ils étaient présents sur toute la périphérie. Les tours mesuraient entre 5,30 et 6,40 m en largeur et 3,60 $\mathrm{m}$ à 3,80 $\mathrm{m}$ de saillie par rapport à la muraille.

35. La tour conservée est visible sur les plans de Plouvier (cf. infra).

36. Enquête du Vicomte d'Avranches, Archives Nationales, Trésor des Chartes, Layettes, J. 240, $\mathrm{n}^{\circ} 18$.

37. Analyses pétrographiques réalisées par X. Savary (Service d'Archéologie du Calvados), et L. Dujardin (univ. Caen).

38. R. PlouvieR, Plan du premier estage de l'abbaie de Landevenec..., Archives nationales, Inv. N III, Finistère 4, pièce $n^{\circ} 4$. M. GERMAIN, "Abbaye de St Guignolé de Landévennec, vers 1680 ", Monasticon Gallicanum, Paris, 1871, pl. 159.

39. Devis des réparations de la mense abbatiale établi par l'ingénieur David, 1782, Archives départementales du Finistère, $100 \mathrm{~J} 267$.

40. Mémorial de Landévennec: manuscrit latin 12703 de la Bibliothèque nationale. Traduction M. MAYMARD, osb.

\section{AUTEURS}

\section{RONAN PÉRENNEC}

Centre départemental de l'archéologie du Finistère, co-directeur des recherches sur l'ancienne abbaye de 1989 à 2001, puis responsable d'opérations préventives et programmées sur différents sites du Finistère

\section{ANNIE BARDEL}

Ancien membre de l'UMR CNRS 6566, responsable des recherches à Landévennec depuis 1978 